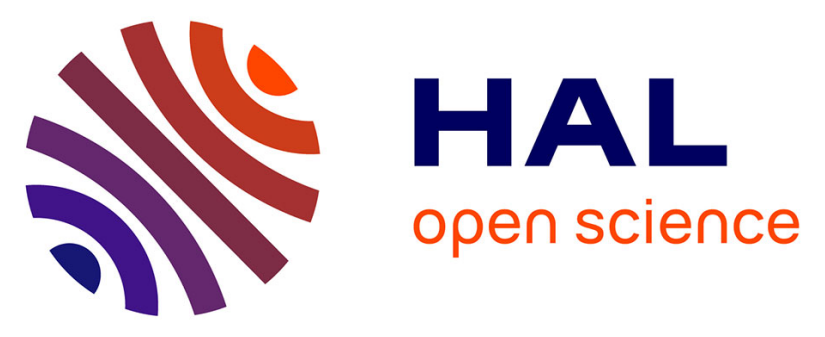

\title{
Implementation of an ontological reasoning to support the guideline-based management of primary breast cancer patients in the DESIREE project
}

Jacques Bouaud, Sylvia Pelayo, Jean-Baptiste Lamy, Coralie Prebet, Charlotte Ngo, Luís Teixeira, Gilles Guezennec, Brigitte Seroussi

\section{To cite this version:}

Jacques Bouaud, Sylvia Pelayo, Jean-Baptiste Lamy, Coralie Prebet, Charlotte Ngo, et al.. Implementation of an ontological reasoning to support the guideline-based management of primary breast cancer patients in the DESIREE project. Artificial Intelligence in Medicine, 2020, 108, pp.101922. 10.1016/j.artmed.2020.101922 . hal-02969742

\section{HAL Id: hal-02969742 \\ https://hal.sorbonne-universite.fr/hal-02969742}

Submitted on 16 Oct 2020

HAL is a multi-disciplinary open access archive for the deposit and dissemination of scientific research documents, whether they are published or not. The documents may come from teaching and research institutions in France or abroad, or from public or private research centers.
L'archive ouverte pluridisciplinaire HAL, est destinée au dépôt et à la diffusion de documents scientifiques de niveau recherche, publiés ou non, émanant des établissements d'enseignement et de recherche français ou étrangers, des laboratoires publics ou privés. 


\title{
Implementation of an ontological reasoning to support the guideline-based management of primary breast cancer patients in the DESIREE project
}

\author{
Jacques Bouaud $^{\mathrm{a}, \mathrm{b}}$, Sylvia Pelayo ${ }^{\mathrm{c}}$, Jean-Baptiste Lamy ${ }^{\mathrm{b}}$, Coralie Prebet ${ }^{\mathrm{d}}$, Charlotte Ngo $^{\mathrm{e}, \mathrm{f}}$, \\ Luis Teixeira ${ }^{\mathrm{f}, g}$, Gilles Guézennec ${ }^{\mathrm{b}}$, Brigitte Séroussi ${ }^{\mathrm{b}, \mathrm{h}, *}$ \\ ${ }^{a}$ Délégation à la recherche clinique et à l'innovation, AP-HP, F-75010 Paris, France \\ ${ }^{\mathrm{b}}$ Sorbonne Université, Université Sorbonne Paris Nord, INSERM, LIMICS UMR_S 1142, F-75006, Paris, France \\ ${ }^{\mathrm{c}}$ Univ. Lille, INSERM, CHU Lille, CIC-IT 1403/Evalab, EA 2694, F-59000, Lille, France \\ ${ }^{\mathrm{d}}$ Service d'oncologie médicale, Hôpital Tenon, AP-HP, F-75020, Paris, France \\ ${ }^{\mathrm{e}}$ Service de chirurgie cancérologique, gynécologique et du sein, Hôpital Européen Georges Pompidou, AP-HP, F-75908, Paris cedex 15, France \\ ${ }^{\mathrm{f}}$ Faculté de santé, Université de Paris, F-75006 Paris, France \\ ${ }^{\mathrm{g}}$ Service d'oncologie médicale, Hôpital Saint-Louis, AP-HP, F-75010 Paris, France \\ ${ }^{\mathrm{h}}$ Département de santé publique, Hôpital Tenon, AP-HP, F-75020, Paris, France
}

\section{A R T I C L E I N F O}

\section{Keywords:}

Clinical decision support systems

Ontology

Clinical practice guidelines

Breast cancer

\begin{abstract}
A B S T R A C T
The DESIREE project has developed a platform offering several complementary therapeutic decision support modules to improve the quality of care for breast cancer patients. All modules are operating consistently with a common breast cancer knowledge model (BCKM) following the generic entity-attribute-value model. The BCKM is formalized as an ontology including both the data model to represent clinical patient information and the termino-ontological model to represent the application domain concepts. This ontological model is used to describe data semantics and to allow for reasoning at different levels of abstraction. We present the guidelinebased decision support module (GL-DSS). Three breast cancer clinical practice guidelines have been formalized as decision rules including evidence levels, conformance levels, and two types of dependency, "refinement" and "complement", used to build complete care plans from the reconciliation of atomic recommendations. The system has been assessed on 138 decisions previously made without the system and re-played with the system after a washout period on simulated tumor boards (TBs) in three pilot sites. When TB clinicians changed their decision after using the GL-DSS, it was for a better decision than the decision made without the system in $75 \%$ of the cases.
\end{abstract}

\section{Introduction}

On the worldwide level, breast cancer is the most common cancer among women and the second most common cancer. In 2018, two million new cases and more than 600,000 deaths were reported. ${ }^{1}$ In France, breast cancer is the most frequent cancer with almost 58,000 new cases per year, in front of prostate cancer, lung cancer, and colorectal cancer with 50,000, 46,000 and 43,000 new cases per year, respectively. After having doubled between 1985 and 2005, breast cancer incidence is globally currently stable in France. More precisely, whereas breast cancer incidence is decreasing for women aged 50-79 who may benefit from the breast cancer national screening program promoted by the National Health Insurance, it is increasing by more than $60 \%$ for women aged $30-49$. With 10,000 deaths per year (consolidated figures in 2018), breast cancer mortality is declining in France and breast cancer is one of the best prognosis cancers with among the best five and ten-year survival rates $(87 \%$ and $76 \%$, respectively). However, it still remains a therapeutic challenge especially for triple negative breast cancers and HER2 + breast cancers, for which improvements are both possible and necessary [1].

Clinical practice guidelines (CPGs) are free-text documents developed by National agencies or academic associations to provide the best recommendations for the management of a set of selected patient profiles. These recommendations are built from published clinical research results and represent the state of the art following evidencebased medicine principles [2,3]. Although studies have shown that implementing oncology CPGs does improve clinical outcomes in both overall and recurrence-free survivals of cancer patients [4-11], there

\footnotetext{
* Corresponding author at: Sorbonne University, LIMICS UMR_S 1142, 15 Rue de l'Ecole de Médecine, 75006, Paris, France.

E-mail address: brigitte.seroussi@aphp.fr (B. Séroussi).

${ }^{1}$ https://gco.iarc.fr/today/data/factsheets/cancers/20-Breast-fact-sheet.pdf
} 
are still variations in clinical practices and the compliance of clinician decisions with CPGs remains low [6,12], e.g., Wöckel et al., reported a $51.9 \%$ compliance rate for the decision of comprehensive care plans for breast cancer patients [6].

In recent years, most countries have implemented organizational measures such as multidisciplinary team meetings or tumor boards (TBs) to promote the shared decision of the various health professionals involved in the management of cancer patients [13]. The goal is to bring together specialists (surgeons, oncologists, radiation therapists, radiologists, pathologists, geneticists, etc.) to discuss each patient case and be able to collectively build the best patient-specific and evidencebased care plan. TBs are also expected to improve CPG implementation as well as clinical trial enrolment. While studies have shown that TBs can improve the compliance of decisions with CPGs [14-16], organization of TBs is hindered by the complexity of discussed patient cases and the amount of information to manage. There is indeed a large number of cases to discuss and finally little time to devote to each of them leading to question the actual impact of TBs on care quality [17-19].

Clinical decision support systems (CDSSs) are health IT tools that require computable biomedical knowledge, person-specific data, and a reasoning or inferencing mechanism that combines knowledge and data to generate and present, at appropriate times, intelligently filtered information to clinicians in order to enhance the quality of their decisions and consequently the quality of the care delivered. While the sole dissemination of free-text CPGs showed to have a low impact on clinician behavior, studies reported that embedding CPGs within CDSSs could improve the compliance of clinician decisions with best practices, in general [20-22] and in the specific case of cancer care decisions made by TB clinicians [23-25].

DESIREE is a European project funded under the H2020 program. The objective is to develop a web-based software ecosystem dedicated to the personalized, collaborative, and multidisciplinary management of primary breast cancer, from diagnosis, to therapy, and follow-up. The DESIREE platform offers some image-based diagnostic decision support modalities involving mammogram-based breast density classification [26], fully automated breast boundary and pectoral muscle segmentation [27], and breast mass classification using ensemble convolutional neural networks [28]. Research works on predictive modeling have also been conducted, e.g., to predict the esthetic outcome of Breast Conservative Therapy considering mechanical forces due to gravity, breast density and tissue distribution, and the inflammation induced by radiotherapy and the wound healing [29]. Additional decision support services have been developed to support the therapeutic decisions of TB clinicians [30]. The first decision support module is based on the proposal of guideline-based recommendations from patient data. Since CPGs have many flaws [31], e.g., they are incomplete, ambiguous, and do not take into account patient preferences, it happens that nonguideline-compliant TB decisions are legitimate. In this case, they have a clinical value that should be capitalized as another source of knowledge, beyond CPGs. Thus, DESIREE offers a second decision support module based on the experience gained from non-compliant TB decisions [32]. Finally, a third decision support module has been developed based on the implementation of a case-based reasoning process where the goal is to reuse past TB decisions made for patients similar to the new patient case discussed by TB clinicians [33].

The three therapeutic decision support modalities have been implemented within the DESIREE platform as three complementary and interoperable decision support modules, denoted GL-DSS for the guideline-based module, EXP-DSS for the experience-based system, and CB-DSS for the one using the case-based reasoning. We used semantic web tools to implement both GL-DSS and EXP-DSS. More specifically, we have built a breast cancer knowledge model (BCKM) as an ontology used as a conceptual and terminological structure federating the three decision support modules for representing knowledge and patient data [34]. This paper is focused on the presentation of the BCKM and the
DESIREE GL-DSS module of the DESIREE platform.

\section{The guideline-based decision support module}

\subsection{Overview of DESIREE components}

The DESIREE platform offers several complementary modalities of decision support [30]. If they differ in the knowledge resources they use and the reasoning process they implement, the three decision support modules are articulated around a common knowledge model, the BCKM, represented as an ontology including both the clinical information model and the termino-ontological aspects of the domain to describe data semantics and allow for reasoning at different levels of abstraction. This requires that patient data that feeds decision support modules be consistent with the BCKM.

On the DESIREE platform, the DESIREE information management system (DESIMS) acts as an electronic patient record where patient data is stored in a dedicated database. Through the user interface, it enables patient data entry and output visualization, as well as the control of all DESIREE components implemented as web services. The outputs of the different components are displayed in the user interface using dedicated display presentations. Internally, patient-related data are provided to the different decision support modules using the FHIR exchange format [35] where DESIMS internal data encoding is transformed into a BCKMconsistent representation. Fig. 1 depicts the main interactions between the three decision support modules.

\subsection{One ontological model to represent both data and knowledge}

The originality of our approach is to represent in the same ontological framework both the clinical information model, i.e., the data model, and the termino-ontological model for the characterization of the application domain concepts. Thus, the BCKM is an explicit specification of all entities and concepts considered as necessary for the management of breast cancer patients. It is a static central resource allowing for interoperability in terms of data structure and semantics. It is also a practical resource to be used by all decision support modules and according to which all data structures and concepts used for the application should be consistently defined. The BCKM is represented as a formal ontology coded in OWL. This allows for the combination of two types of inferences: subsumption-based inferences, i.e., ontological reasoning based on description logic (DL), and arbitrary application of domain-dependent deductions based on production rules.

The structuring of the BCKM ontology replicates the entity-attribute-value (EAV) generic model for data modeling and the integration of concepts related to the breast cancer domain [34]. Thus, the BCKM is not intended to constitute a reference ontology that would cover all aspects of breast cancer, but it rather gathers the body of knowledge required for the computer-based therapeutic management of breast cancer patients. This involves allowing for (i) the representation of patient data collected using an electronic patient record developed outside the DESIREE project, as well as (ii) the formalization and the operationalization of the knowledge embedded within CPGs to provide decision support.

\subsubsection{Ontological representation of the EAV data model}

Unlike information models dedicated to the biomedical field that offer specific predefined objects for hospital information systems or electronic health records (OpenEHR, FHIR...), the EAV model is a generic model, considered flexible enough to model biomedical data [36-38]. From a logical point of view, data models, whether relational or object-oriented, can be translated into the EAV model. We therefore structured the ontology through the prism of EAV model components and chose to explicitly represent the three elements of the EAV model as classes: entities, attributes, and values. 


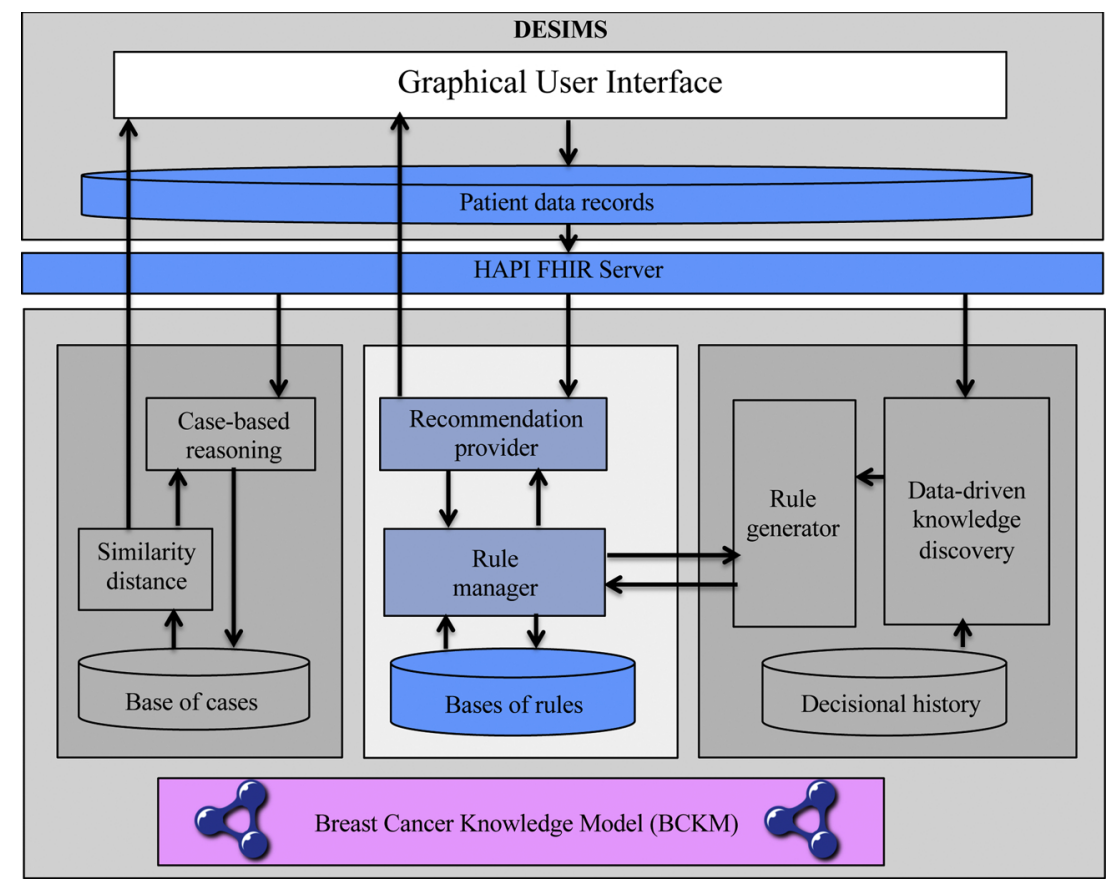

Fig. 1. General architecture and information flows between the different DESIREE decision support modules and the DESIMS.

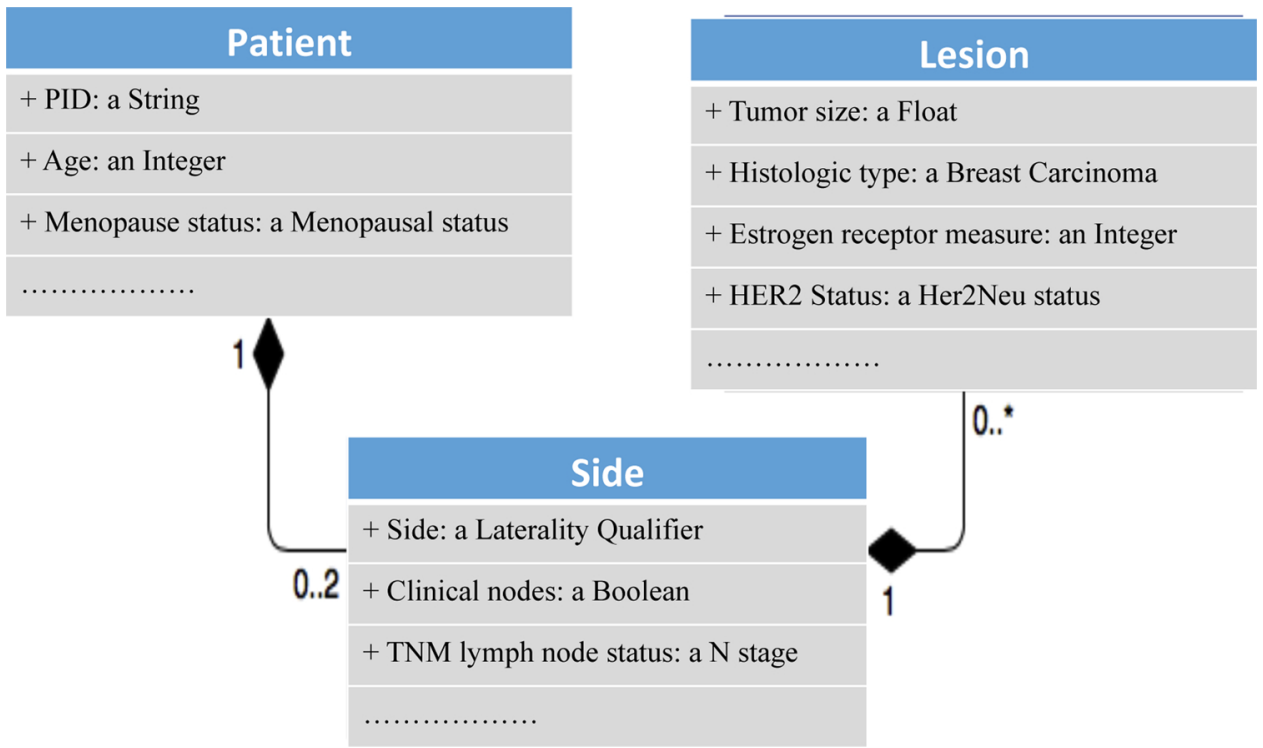

Fig. 2. Excerpt from the UML class diagram representing the three main clinical entities (Patient, Side, and Lesion) used to describe a breast cancer clinical case, and their relationships.

- ModelEntity subclasses are used to define the components of the data model. In our application, they correspond to entities that describe a patient case and are relevant in the decision-making process, e.g., anatomical entities to characterize the disease, the patient (PatientEntity), the side (SideEntity), and the lesion (LesionEntity) (see Fig. 2). Other entities are associated to the patient and characterize her context such as her relatives, prior treatments, and examinations carried out, but also the outcomes issued by the decision support module like recommended care plans or alert messages.

- ModelAttribute subclasses list the attributes of different entities, e.g., the age for the patient (Age), the presence of lymphadenopathies for a side (ClinicalLymphNodes), or the histological type of a lesion (HistologicType). Internally, each class of an attribute is declared to belong to an entity using the object property isAttributeOf.
- ModelValue subclasses represent the different value types declared for attributes. These subclasses correspond to classic primitive types such as integers, floats, booleans, dates, strings. HierarchicalValue is a separate subclass which subclasses are made of discrete value sets structured as hierarchies organized by the subsumption relation. For instance, the two sex values, male and female, are grouped in a flat set of exclusive values, but the types of breast cancer (BreastCarcinoma) are described hierarchically by the set of invasive cancer, in situ cancer, and Paget's disease, each one being refined by more specific subclasses. The interest of these subsumption-structured sets of values is to allow the collection of information at different levels of abstraction and to reason at these different levels. In the ontology, the specification of the value type of an attribute is done by the object property hasRange linking the attribute class and the class of the value type, e.g., the Age attribute of a 
patient is linked by hasRange to IntegerValue, the ClinicalLymphNodes attribute to BooleanValue, and the HistologicType attribute of a lesion to the BreastCarcinoma class.

- Relationships between entities are represented by object properties between classes of entities. For instance, a LesionEntity is linked to the SideEntity by the object property hasSide. These data model relationships are sub-object properties of the isRelatedTo object property. This allows for distinguishing between internal object properties and the relationships specific to the data model.

\subsubsection{Building the breast cancer knowledge model}

Within the DESIREE project, one of the first tasks was to build the application domain model. The elicitation of relevant data elements and their collection were done by combining the expertise of different project partners, such as breast cancer clinicians, electronic health record software developers, guidelines and knowledge representation experts. Among the collected data elements, a subset was referenced as the minimum data set necessary to enable state-of-the-art decisionmaking in the management of primary breast cancer. This information has been formalized to comply with the BCKM core framework presented above (2.2.1). Four periods of interest or "scenarios" have been identified in the breast cancer management clinical pathway: scenario A when the cancer has just been diagnosed and the decision may be surgery or neoadjuvant therapy, scenario B when a neoadjuvant therapy has been administered and TB clinicians have to decide the surgery or the radiotherapy protocol if the patient is non-operable, scenario $\mathrm{C}$ when neoadjuvant therapy and surgery have been administered, and scenario D when surgery has been first performed and adjuvant treatment modalities have to be decided.

The set of concepts related to the application domain is represented as subclasses of the core concepts. Among the introduced concepts, we identified those corresponding to the entities of the data model, such as the patient, the sides, the lesions, the procedures, and the recommendations. We also identified the different attributes characterizing each entity (e.g., age, histological type, tumor size, etc.). Finally, the set of hierarchical values specific to the application domain was integrated into the ontology. As previously said, these sets include both unstructured sets of simple values (sex with male and female values, grade with low grade, intermediate grade, and high grade), and sets of values hierarchically structured by subsumption offering different characterization levels (histological types of breast cancer, TNM classification, breast cancer stages, and all the different therapeutic procedures like surgeries, chemotherapies, and so on). To collect domainspecific value sets, we reused available existing resources, e.g., the National Cancer Institute thesaurus (NCI thesaurus, or NCIt). The NCIt provides a reference terminology for many aspects of cancer management: "it covers vocabulary for clinical care, translational and basic research, and public information and administrative activities." ${ }^{2}$. It includes for each concept textual definitions, several synonyms, relationships with other NCIt concepts including subsumption relationships, and mappings with concepts in other resources like SNOMED CT. Moreover, it is available as an OWL ontology so that some concepts or subclass hierarchies can be reused. Finally, using reference terminologies to build the BCKM is a step towards semantic interoperability with external data sources. On the technical side, we used a tabular ontology tool, named Flat OWL Editor [39], to populate the BCKM. The tool allows to extract concepts and sub-hierarchies from existing ontologies, edit them when necessary, and then insert the updated or new additional sub-hierarchies into the BCKM.

Fig. 3 illustrates the organization of the resulting ontology by distinguishing the core ontology, dedicated to the data model, and the subhierarchies of domain concepts stricto sensu.

Fig. 4 displays an extract of the BCKM in Protégé, a popular Open

\footnotetext{
${ }^{2}$ https://ncithesaurus.nci.nih.gov/ncitbrowser/
}

Source OWL Editor [40]. For instance, the concept HistologicType is an attribute (isAttributeOf) of the SideEntity entity and has a value type (hasRange) BreastCarcinoma. The HistologicType concept exists in the NCIt and the information originating from the NCIt is recorded as annotations, like the NCI label, the NCI code, the NCI definition, or the UMLS CUI. Fig. 4 also shows that BreastCarcinoma is a subclass of HierarchicalValue and has subclasses structured as a hierarchy.

\subsection{Rule-based knowledge representation}

\subsubsection{A data model-driven rule language}

We used the Natural Rule Language (NRL) [41] as the formalism to specify rules in order to represent guideline knowledge. The IF part of rules checks various constraints on the values of different attributes of the selected entities, including equality tests, numerical comparisons, or subsumption checking with the "is a kind of" operator. Specific logical constructs allow for the negation in rules by checking the existence of complex expressions. The THEN part of rules contains actions that build recommendations (the model of which is described in Section 2.3.2). Fig. 5 provides an example of an NRL decision rule. This rule matches two entities, a side (theSide) of a patient (thePatient). The THEN part is made of the disjunction of two recommended actions. Other NRL constructs in the THEN part allow for the creation of data model components (see Fig. 9).

\subsubsection{The recommendation model}

The therapeutic management of breast cancer patients is made of a care plan including one or several actions to be performed synchronously or in an ordered sequence. These actions may correspond to different therapeutic modalities (surgery, oncology protocols, radiotherapy plans, etc.) or to any other relevant action like examination, surveillance, referral to a specialist, etc., e.g., 'mastectomy and axillary lymph node dissection' is a care plan made of two synchronous actions, 'systemic chemotherapy, then radiotherapy and endocrine therapy' is a care plan made of sequential actions. Moreover, when a care plan is decided by TB clinicians, the different actions may be described at different levels of abstraction, for instance 'endocrine therapy' or, more specifically, 'tamoxifen therapy', depending on the information available at the moment the clinical case is discussed.

In DESIREE, a recommendation is formally specified in the BCKM and is represented as a care plan. A Recommendation (RecoEntity) is composed of one or several orders (OrderEntity), and each order is linked to one action (ActionEntity). An action is mainly characterized by a BreastCancerProcedure which is the super class for the hierarchy of breast cancer procedures in the BCKM. In addition to the action, an order is characterized by several important attributes or relationships as displayed in Fig. 5:

- The step to specify the rank of the action in the ordered sequence. In Fig. 5 , the step is ' 1 ' to represent the first action of a sequence.

- The entity on which the action has to be performed, for instance a chemotherapy is performed on the patient, whereas mastectomy and re-excision are performed on the side.

- The evidence level of the action as provided in CPGs. For instance, NCCN guidelines propose categories of evidence where the highest level is 1 , the default is $2 \mathrm{~A}$, and the lowest is 3 [42].

- The conformance level to indicate what is the expected conformance of end-users with the action. We adopted a classical qualitative sixvalue scale with SHALL when the action is required, SHOULD when the action is recommended, MAY when the action is possible, along with their negative counterparts SHALLNOT, SHOULDNOT, and MAYNOT.

As previously said, clinical knowledge, including guideline knowledge, can be described at varying levels of abstraction based on the information available on a patient. For example, CPGs recommend that 


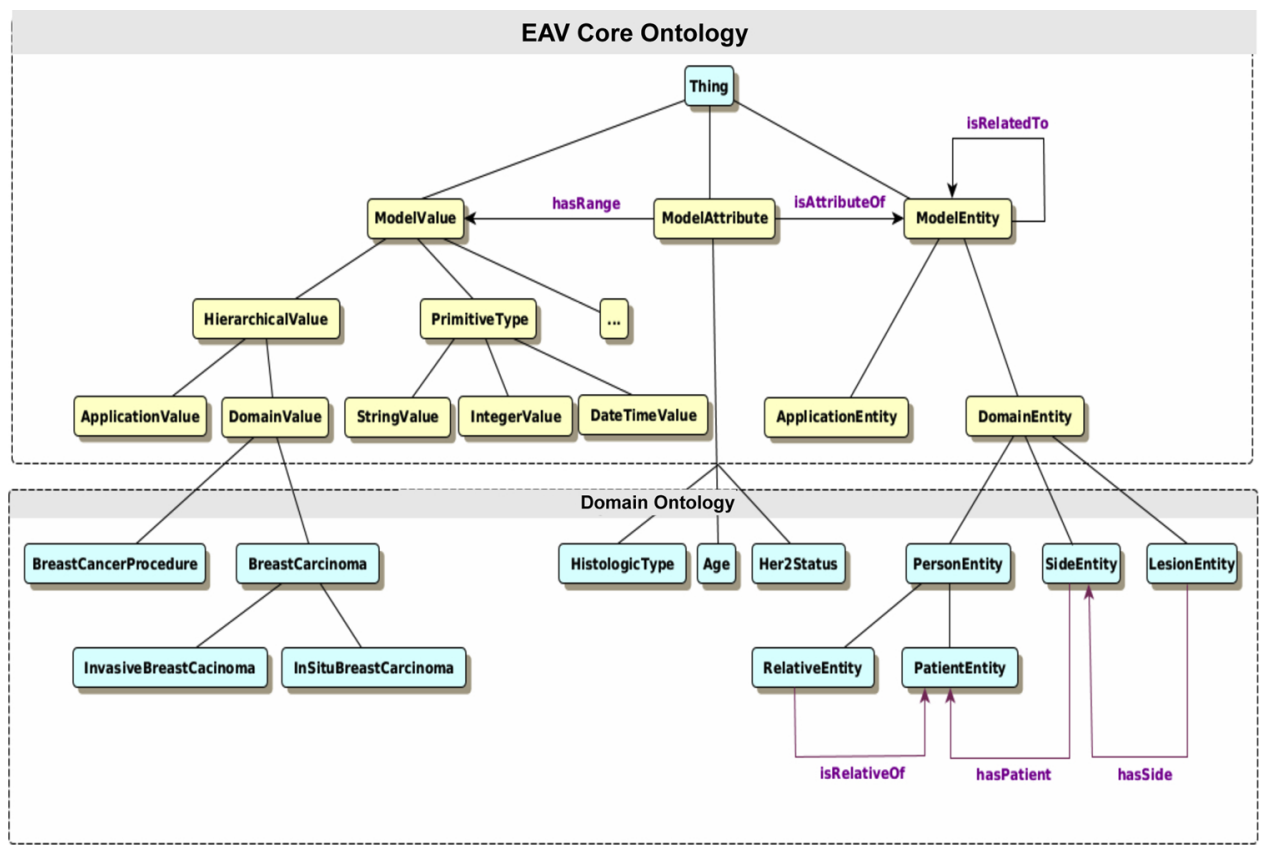

Fig. 3. Structuration of the top of the core ontology with domain concepts integrated at the bottom.

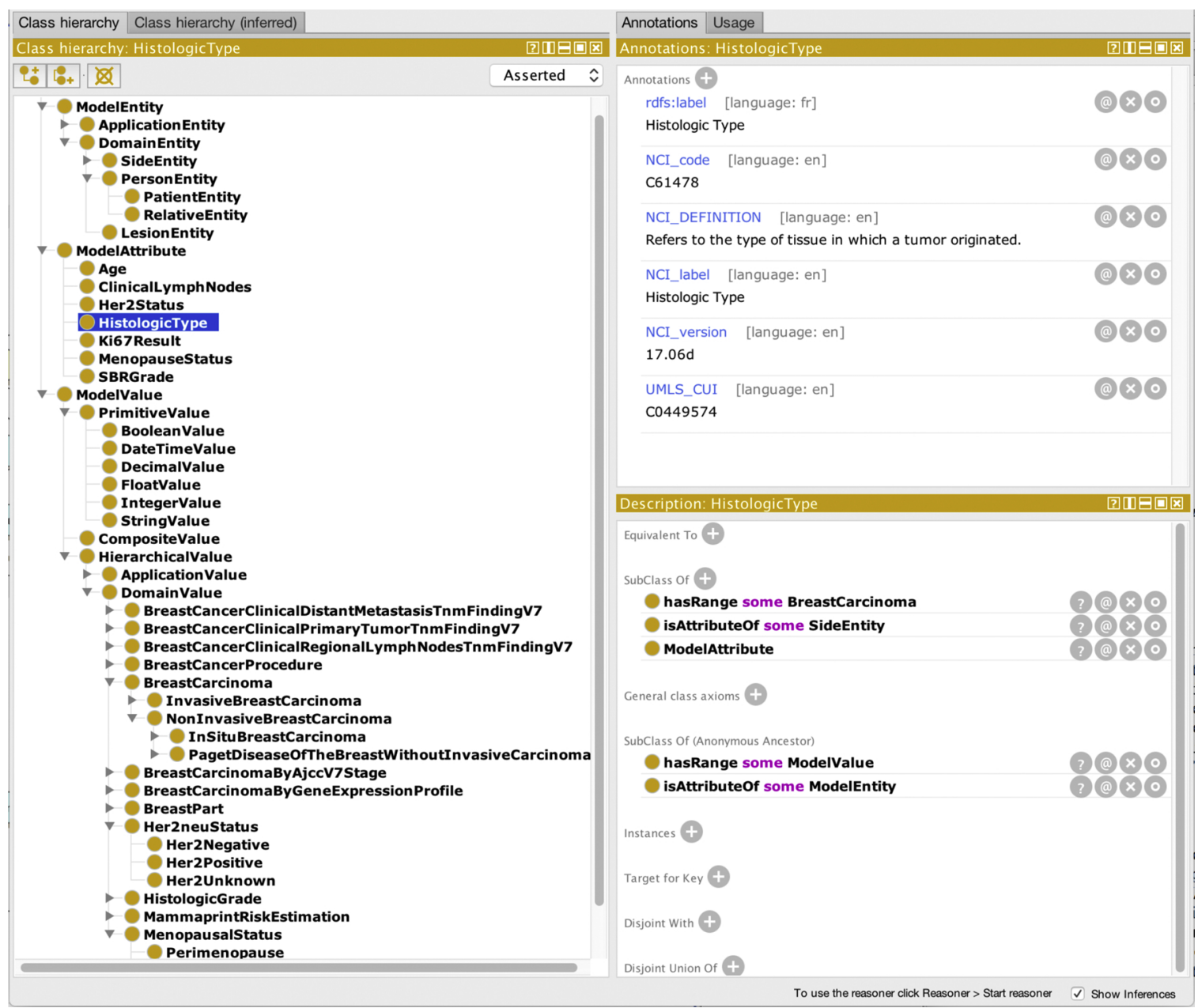

Fig. 4. Extract of the BCKM ontology in Protégé. 


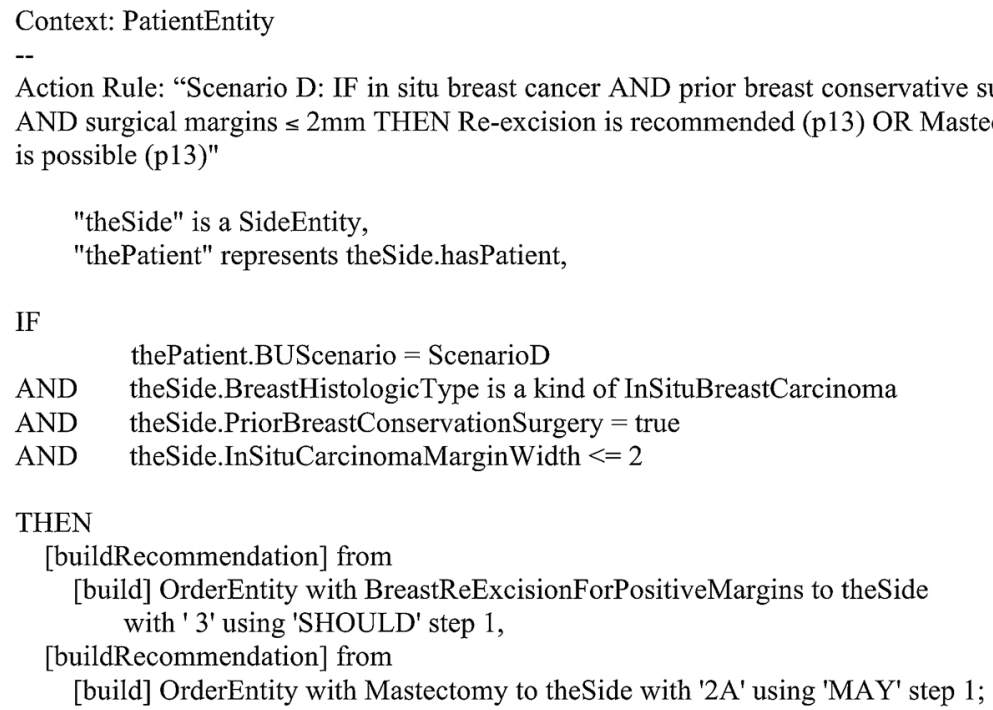

Fig. 5. Example of an NRL decision rule to exemplify the consistency with the BCKM.

Guideline used: NCCN (exp=false)

\section{Lumpectomy + ClipsOnTumorBed}

\section{BreastCancerSystemicTherapy}

$>$ SystemicChemotherapy

$>$ Paclitaxel

\section{> Doxorubicin Cyclophosphamide}

Fig. 6. Illustration of complement and refinement dependencies between recommendations.

a surgical axillary exploration be performed for invasive breast cancers. If axillary lymph nodes are not invaded, the surgical axillary exploration is a sentinel lymph node biopsy, otherwise it is an axillary lymph nodes dissection. Formal rules have been built following the same pattern. We have proposed to enrich recommendations produced under a given context by the provision of two types of dependencies between an order and a new recommendation, a "refinement" relationship and a "complement" relationship. In the first case, the new recommendation includes an order with a more specific action, whereas in the second case, the action is complemented by another recommendation. Fig. 6 illustrates the two dependencies, with the example of lumpectomy complemented (noted with a ' +') with clips on the tumor bed, and the systemic therapy refined as a systemic chemotherapy, itself refined by two chemo protocols, paclitaxel and doxorubicin-cyclophosphamide.

Two operators have been added in the THEN part of the rule language to create recommendations that depend on an order of a recommendation (AddComplementReco and AddRefinementReco). Fig. 7 provides two rules used to generate some dependent recommendations according to the two variants of the example provided in Fig. 6 .

\subsection{Clinical practice guidelines modeling}

\subsubsection{Formalization of CPG knowledge}

The preliminary step was to select international breast cancer management CPGs to cover the worldwide state of the art as well as the "local guidelines" currently implemented by DESIREE clinical partners.
This step resulted in the selection of several CPGs among which three were encoded in an executable format: NCCN@ guidelines published by the network of 27 cancer centers in the United States [42], internationally considered as reference guidelines, and two local guidelines, the French recommendations published by the Assistance Publique Hôpitaux de Paris (AP-HP) [43], and the recommendations published by Onkologikoa, both being clinical partners of the DESIREE project. Thus, we encoded the three CPGs to be used as knowledge bases for decision support, with (i) the chance to reduce the decision support silence when CPGs are complementary and some CPGs may provide recommendations to fill in the knowledge gaps of the others, and (ii) the risk to increase decisional conflicts when CPGs are inconsistent and recommendations provided by the ones are in contradiction with recommendations provided by the others.

In a way similar to the DeGel approach [44], the translation from free-text CPGs to computer-interpretable guidelines was performed in several steps, from free text (original CPGs), to semi-structured text, semi-formal text, and a formal, machine-executable representation. These different steps are listed below using the example of re-excision in the case of an in-situ breast cancer as displayed in Fig. 8.

- The first step aims at building a human-readable semi-structured version of CPG contents as IF-THEN statements keeping the reference to where the recommendation was quoted in free-text guidelines, the evidence level (EL) when available, and the expected conformance level. This step was performed by a medical oncologist specialized in the management of breast cancer (CP). Then, rules were validated by clinical partners (CN, LT). In the example displayed in Fig. 8, we obtained:

"IF In situ breast cancer AND prior Tumorectomy performed AND there is a lesion with margins smaller than $2 \mathrm{~mm}$ THEN re-excision by Tumorectomy is recommended ( $\mathrm{EL}=3, \mathrm{p} 13)$ OR Mastectomy is possible $(\mathrm{EL}=2 \mathrm{~A}, \mathrm{p} 13)$ "

- The second step is based on the completion and standardization of the notions used in the rules according to concepts defined in the knowledge model to reach a semi-formal version of CPGs. This led to rewriting the rules in a pseudo-logical language that remained nevertheless understandable by clinicians. This step was performed by a medical computer scientist with a good knowledge of the management of breast cancer (BS). The previous statement was rewritten as follows with BCKM concepts: 


\begin{tabular}{|c|c|}
\hline $\begin{array}{l}\text { Context: PatientEntity } \\
-- \\
\text { Action Rule "Example of complement" }\end{array}$ & $\begin{array}{l}\text { Context: PatientEntity } \\
-- \\
\text { Action Rule "Example of refinement" }\end{array}$ \\
\hline $\begin{array}{l}\text { "theLesion" is a LesionEntity, } \\
\text { "theAction" is a ActionEntity, } \\
\text { "theOrder" represents theAction.hasOrder, }\end{array}$ & $\begin{array}{l}\text { "thePatient" is a PatientEntity, } \\
\text { "theAction" is a ActionEntity, } \\
\text { "theOrder" represents theAction.hasOrder, }\end{array}$ \\
\hline IF $\quad$ thePatient.BUScenario $=$ ScenarioAC & IF $\quad$ thePatient.BUScenario $=$ Scenario $A D$ \\
\hline AND theOrder.hasObject $=$ theLesion & AND theOrder .hasObject $=$ thePatient \\
\hline AND theAction $\cdot$ hasOrder $=$ theOrder & AND theAction $\cdot$ hasOrder $=$ theOrder \\
\hline AND theAction .Action = Lumpectomy & AND theAction .Action $=$ BreastCancerSystemicTherapy \\
\hline AND theOrder.Conformance is one of 'SHOULD', 'MAY' & AND theOrder.Conformance is one of 'SHOULD', 'MAY' \\
\hline $\begin{array}{l}\text { THEN } \\
\text { [AddComplementReco] theOrder from } \\
\text { [build] OrderEntity with ClipsOnTumorBed } \\
\text { to theLesion with ' } 2 \mathrm{~A}^{\prime} \text { ' using 'SHOULD' step 1; }\end{array}$ & $\begin{array}{l}\text { THEN } \\
\text { [AddRefinementReco] theOrder from } \\
\text { [build] OrderEntity with SystemicChemotherapy } \\
\text { to thePatient with ' } 2 \mathrm{~A}^{\prime} \text { ' using 'SHOULD' step 1; }\end{array}$ \\
\hline
\end{tabular}

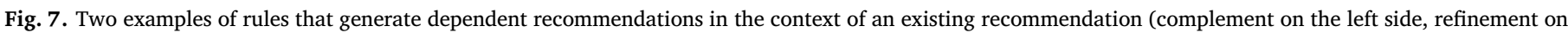
the right side).

"IF InSituBreastCarcinoma AND PriorBreastConservationSurgery AND InSituCarcinomaMarginWidth $<=2$ THEN BreastReExcisionFor PositiveMargins using 'SHOULD' with 3 OR Mastectomy using 'MAY' with $2 \mathrm{~A} "$

- The third and last step aims at encoding the rule in a way that guarantees that the rule description is consistent with the BCKM, the NRL syntax, and DESIREE workflow. This requires to identify the relevant attributes and entities for describing the IF part of the rule and to check the correct object argument for specifying the recommended actions. The resulting formal NRL rule encoding the recommendation presented in the first two steps is the rule given in Fig. 5. A condition about the patient scenario has been added (thePatient.BUScenario = ScenarioD). The other conditions are expressed with attributes of the side entity. For instance, the BreastHistologicType attribute indicates the histologic type of the cancer at the side level, an information which is not provided from raw patient data but was inferred from the anatomopathological description of all lesions in the side (in case of multiple lesions). This pattern is used in many rules to synthetize or abstract information which is required at a high level of abstraction to match guideline knowledge but not directly available in raw patient data. Fig. 9 illustrates a rule that performs abstraction at the side level of raw data given at the lesion level. Moreover, it exemplifies the use of existential quantifiers. The rule reads: "IF the histologic type at the side level is not known, AND there exists at least one lesion of the same side which histologic type is a kind of in situ cancer, AND there is no lesion of the same side which histologic type would be invasive cancer, THEN the histologic type at the side level is in situ cancer".

\subsubsection{Structure and organization of rule bases}

For each CPGs, e.g., NCCN CPGs, AP-HP CPGs, or ONK CPGs, we have built a structured knowledge base formalized as NRL rules. If the NRL syntax allows for grouping sets of rules, this aims at structuring the authoring process and has no impact on rules execution. However, we needed to distinguish two kinds of rules, which implicitly yields two subsets: a set of generic rules, independent from CPGs, and a set of

\section{C - Surgical re-excision indication}

- Margins

- In situ cancer, surgical margins $\leq 2 \mathrm{~mm}$

- Invasive cancer: unclear inked margins

All indications for re-excision must be discussed in tumor boards. guideline-specific rules.

Generic rules describe common knowledge of the breast cancer domain. Most of these rules abstract or synthesize information from raw data at the same entity level, for instance by inferring a new categorial value for an attribute from an existing numerical value of another attribute of the same entity, or by inferring a new entity-attribute-value triple from a more complex situation involving different entities as exemplified in the rule displayed in Fig. 9. The role of these rules is to infer a synthetic representation of the clinical case to enable guidelinespecific knowledge to be triggered. This is especially used in the case of entities linked with a part-of relationship, like a lesion, part of a side, part of a patient, to raise the information from one level to the upper ones, e.g., raise the in situ histologic type of a lesion to the in situ histologic type of a side (if there is no other invasive lesion in the side) (see Fig. 9). Such rules are necessary to properly handle the cases of multifocal cancers (with multiple lesions in the same side) and/or of bilateral cancers (when both sides have the disease). These common rules can be used with the rule bases that represent the different CPGs.

On the contrary, guideline-specific rules encode specific guideline contents and mostly generate recommendations when conditions expressed on the synthetic clinical case are satisfied, as the rule in Fig. 5 exemplifies: "theSide.BreastHistologicType is a kind of InSituBreastCarcinoma AND theSide.PriorBreastConservationSurgery = true AND theSide.InSituCarcinomaMarginWidth $<=2$ " means that the patient is suffering from an in situ breast cancer (there is no invasive lesion) managed by a breast conservative surgery and that there is at least a margin lower than $2 \mathrm{~cm}$ (or exactly one if there is only one lesion). In this case, according to AP-HP CPGs, surgical re-excision is recommended, mastectomy is possible.

\subsection{Implementation}

\subsubsection{Execution engine}

Combining ontological reasoning and custom rules has been studied for long (e.g., [45]) and there exist many tools that enables the two types of inferences with different approaches. In our case, since the rules are written in NRL, one requirement was to be able to implement the source NRL constructs we used in the destination rule language of

Fig. 8. Excerpt from the AP-HP textual CPGs - March 2016, p. 13 (translated) 


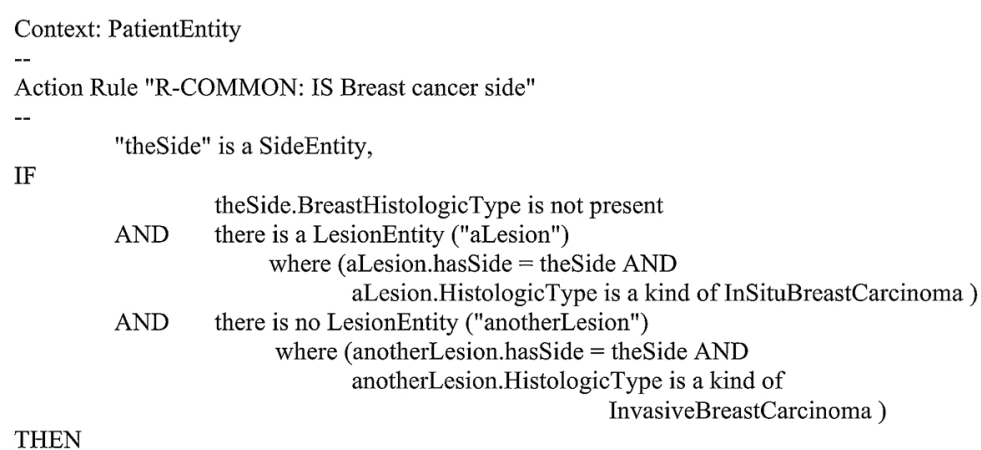

set theSide.BreastHistologicType to InSituBreastCarcinoma;

Fig. 9. Example of a rule abstracting information: the histologic type is abstracted at the side level from data given at the lesion level.

the tool. Basically, rule-based reasoning relies on the closed world assumption where negation is treated by negation as failure. This is not the case with pure DL-based reasoning based on the open world assumption. We needed to use both reasoning types to deal with rules and data as required by the project. Especially, the first type was required to handle an NRL operator like "there is no", as used in the rule displayed in Fig. 9. We chose to use the EYE semantic reasoner [46] which, unlike SWRL, allows constructs like "not exist" or "for all". EYE is written in Prolog and is able to deal natively with triples in the N3 notation [47]. Data and rules are provided to EYE in N3. Considering ontological reasoning, the OWL DL semantics in EYE is managed by explicit rules which are executed together with custom rules in an homogeneous integration framework. Moreover, according to the authors, EYE is reported to be quite efficient on several benchmarks.

Using EYE as the execution engine for the GL-DSS required that patient data, NRL rules, and the BCKM be expressed in N3 to feed the engine. N3 being through the Turtle syntax just another serialization format for the OWL ontology, transforming the BCKM in N3 was quite straightforward. However, the transformations of data and rules required dedicated and consistent parsing. From the user point of view, NRL custom rules corresponding to some given CPGs are executed in a forward-chaining mode by saturation and generate patient-centered recommendations.

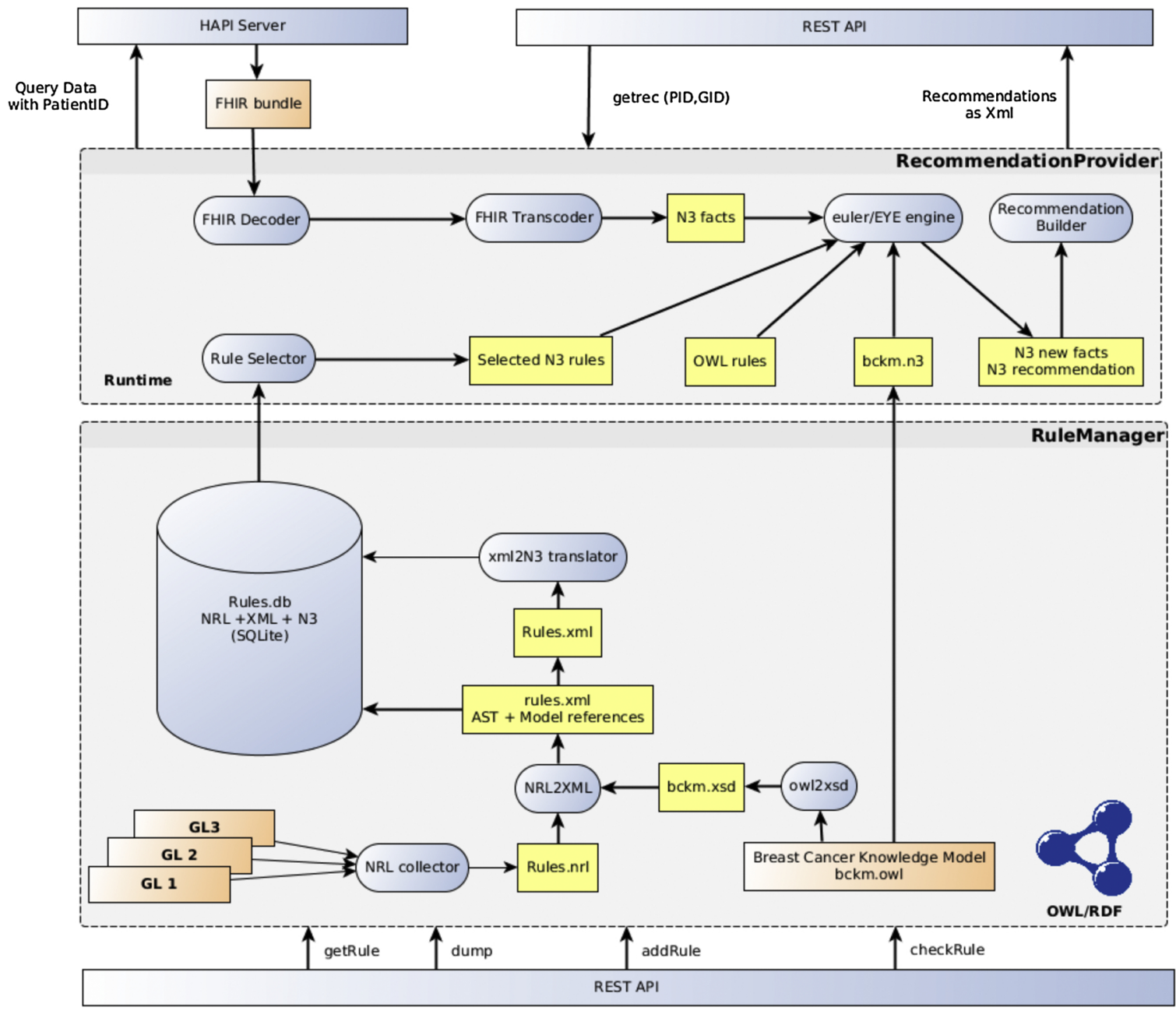

Fig. 10. General scheme and visualization of knowledge and data flows within the GL-DSS engine. 
Quick View - status · eVolution · decision

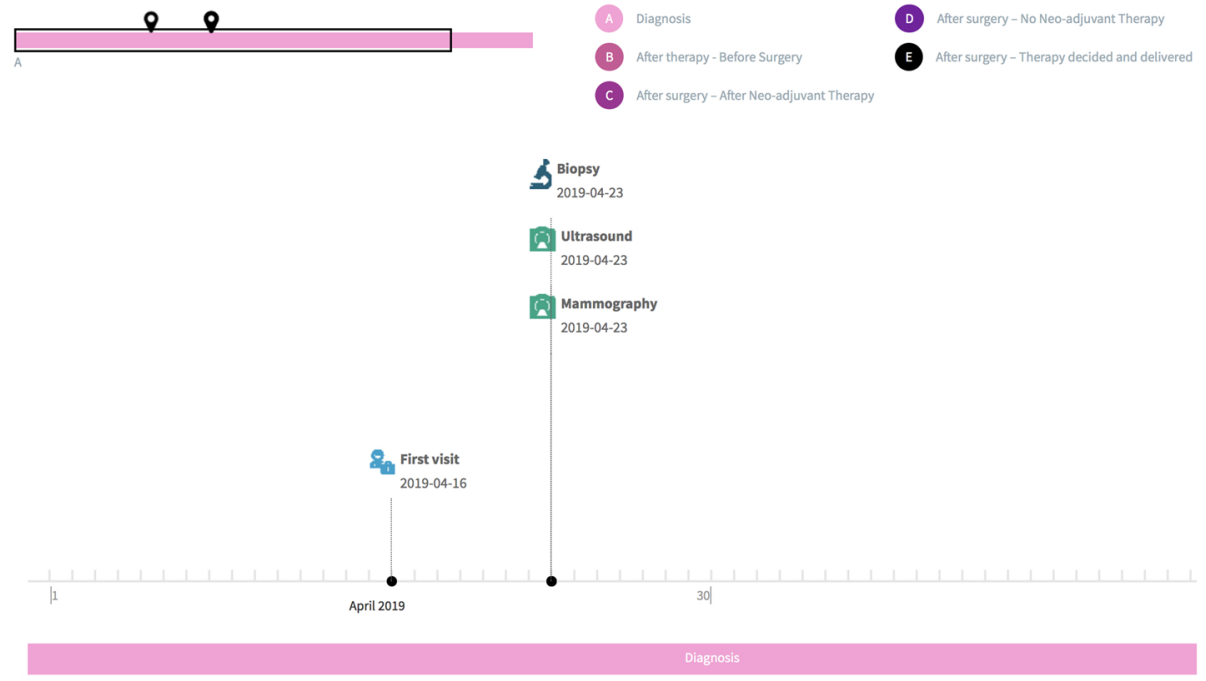

Fig. 11. Time-line display of the different events of the patient clinical pathway.

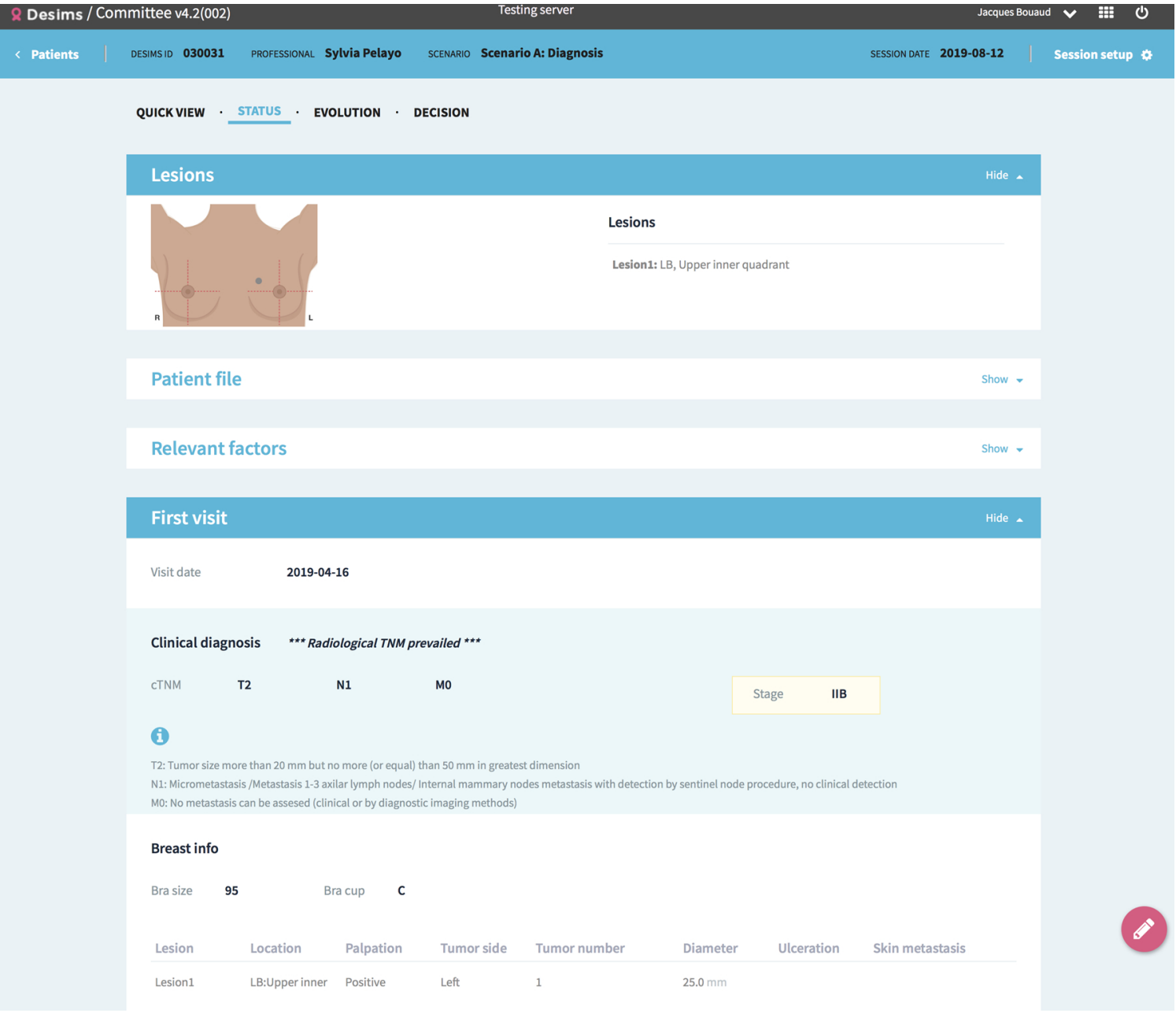

Fig. 12. Screenshot of DESIMS showing a summary of patient data. 


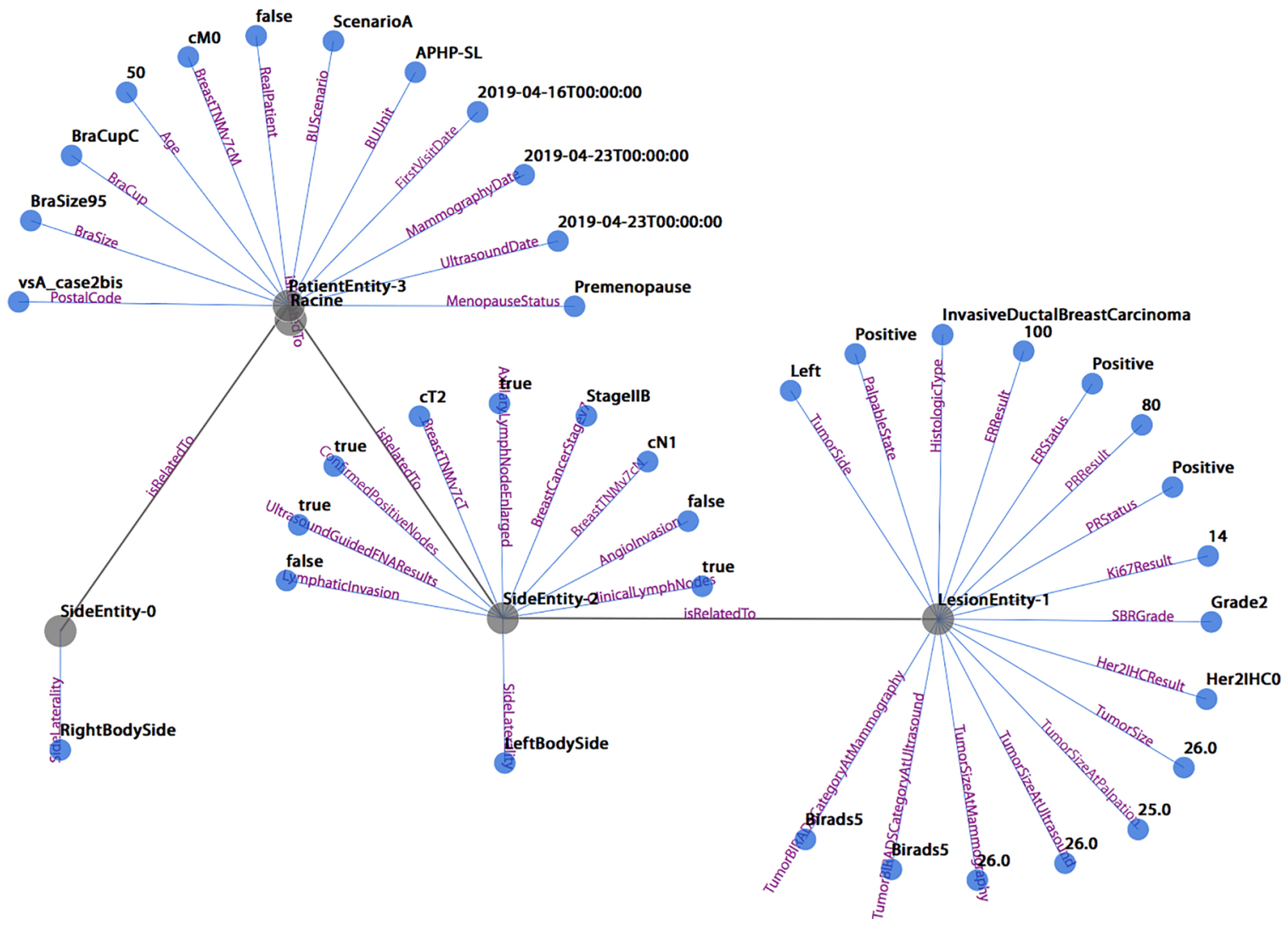

Fig. 13. Graph-based representation of the instantiated data model for the described clinical case.

\subsubsection{Rule management}

A rule manager component has been developed to handle and store the different rule bases. A rule base usually corresponds to the formal version of CPGs, but in DESIREE, a rule base could also originate from the experience-based decision support (EXP-DSS) that learns rules from decisions made in a given clinical site that do not comply with CPGs [32]. Fig. 10 provides a schema of the rule manager workflow. Since NRL is a data model-driven language, the NRL parser requires an explicit data model specification to check the syntax of NRL rules. This reference data model is an XSD model which is automatically derived from the BCKM. The parser can then validate the rules when they are consistent with the BCKM. Once correctly parsed, rules are transformed into an intermediate XML representation similar to the Knowledge Artifacts specification proposed by the HL7 organization [48]. The principle promoted by this approach is to propose a shared knowledge representation independent of the source rule expression language (NRL in our case) and independent of the rule execution language (N3 in our case). The XML representation is also used to exchange rules with the EXP-DSS which generates EXP-based rules in this format that are stored in the rule manager [32]. Finally, the XML rule representation is transformed into N3, the target internal representation of rules, in order to be executed by EYE. The rule manager is implemented as a web service, a tomcat servlet, and its internal rule repository is a basic SQLite database.

\subsubsection{Generation of recommendations}

The recommendation provider is the main component of the GL-DSS and its role is to deliver the recommendations issued for a given patient case following some given CPGs. The recommendation provider is implemented as a tomcat servlet. Data flows generating guideline recommendations are displayed in Fig. 10.

Patient data is stored in the DESIMS in a proprietary database. For interoperability reasons, the DESIMS provides patient data to the different decision support modules through FHIR bundles using a limited number of FHIR resources (Patient, Observation, Body Site, Specimen, Careplan, etc.). For a given patient, all patient-related entities, their attributes, and their values are transferred with a coding scheme consistent with the BCKM in a FHIR message which is stored temporarily on a HAPI FHIR server. The semantic interoperability between the DESIMS and the BCKM is implemented through DESIMS-BCKM code mappings. When the recommendation provider is called with a patient ID, a FHIR decoder is responsible for fetching and parsing the patient's bundle from the HAPI FHIR server. Then the FHIR transcoder translates patient data into N3 triples consistent with the BCKM organization.

From the guideline ID provided as an argument to the recommendation provider call, a rule selector requests the rule manager for the corresponding rule base in the N3 format. Depending on the call arguments, different guidelines can be used (NCCN, AP-HP, or ONK), as well as different sets of experience-based rules. Then the inference engine is executed using these selected N3 rules, the N3 representation of the patient, the N3 representation of the BCKM, and the OWL DL rules provided by EYE.

As a result, the engine produces a set of new triples inferred by the application of the selected rules. This set of triples is transferred to the recommendation builder which extracts from the triple graph the list of structured recommendations as described in 2.3.2 and generates an XML output which, besides the recommendations, also includes the list of triggered rules and the new inferred facts. This output is then returned to the caller, in practice the DESIMS. The whole process is described with a clinical case in the next section. 


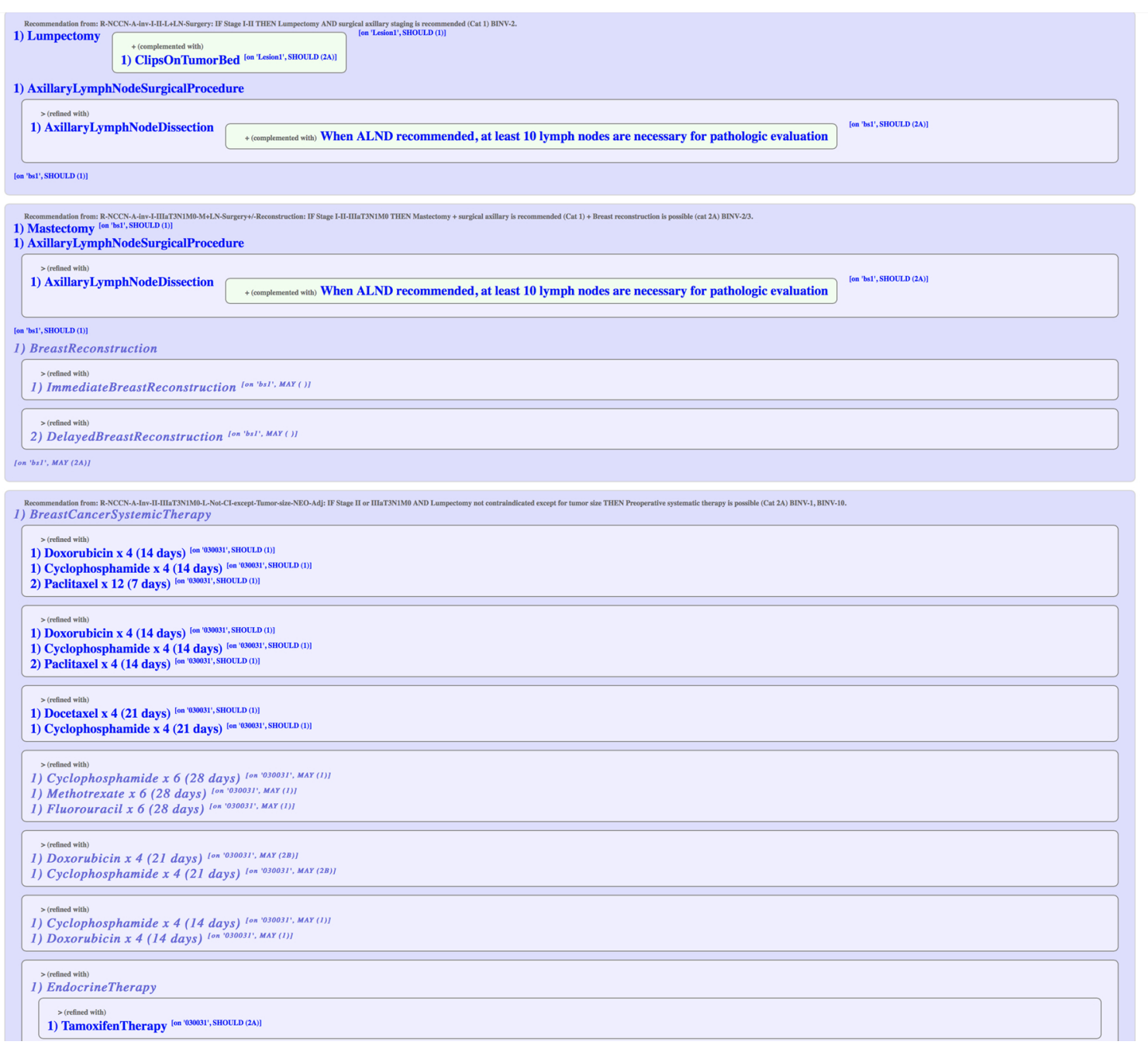

Fig. 14. Visualization of the recommendations produced by the GL-DSS for the described clinical case.

\section{Results}

\subsection{Qualitative and quantitative information about the BCKM and the rule} bases

In the current state, the conceptual model built for DESIREE relies on 22 entities and a total of 394 attributes. Attributes are distributed according to their value type as follows: $49 \%$ Booleans, $9 \%$ integers, 4 $\%$ floats, $5 \%$ strings, $4 \%$ dates, and $33 \%$ refer to hierarchical values. The resulting BCKM ontology contains 1445 classes, 2305 axioms, 25 object properties, 15 data properties. A total of 658 classes are derived from the NCI thesaurus.

NCCN CPGs for breast cancer are among the most utilized comprehensive breast cancer guidelines. The 2017 version of NCCN CPGs is made of a comprehensive document of 199 pages, 75 pages of "blocks" describing decisional algorithms, and 124 pages of narrative guidelines. The more recent breast cancer CPGs from AP-HP (France) have been published in 2016 as a 36 page-long document describing both diagnostic and therapeutic recommendations making the difference between surgery, chemotherapy, and endocrine therapy procedures. Onkologikoa CPGs appear as a set of eight blocks displaying the recommended management of the most frequent breast cancer presentations. The three CPGs have been first structured as a set of humanreadable decision rules, then encoded in NRL. The whole process resulted in three rule bases made of a total of 386 rules for NCCN CPGs, 305 for AP-HP CPGs, and 494 for ONK CPGs. Rule bases share the same subset of common generic rules made of 12 rules.

\subsection{Using DESIREE on a clinical case}

\subsubsection{Case description and data entry}

We consider the case of a 50-year-old woman, in pre-menopause, with a left breast lesion of $25 \mathrm{~mm}$ at palpation and a left axillary lymphadenopathy. Mammography has revealed a lesion in the upper inner quadrant of the left breast, $26 \mathrm{~mm}$, BI-RADS 5. Ultrasound confirmed a $26 \mathrm{~mm}$ lesion, the biopsy of which diagnosed a ductal invasive carcinoma, grade 2 , estrogen receptor $100 \%$, progesterone receptor 80 $\%$, HER2 negative, Ki67 at $14 \%$. The fine needle aspiration of the axillary lymph node retrieved positive tumor cells. Cancer is classified as cT2N1M0, stage IIB. Data has been entered using DESIMS, (the EHR of the DESIREE project). The different procedures already performed can be visualized on a time line as depicted on Fig. 11 .

Fig. 12 shows a screen shot of the DESIMS interface summarizing the status of the patient with a focus on the most important data to be reminded before decision is made by TB clinicians.

Fig. 13 shows the graph based on the triple representation of the instantiated data model that describes the clinical case and which is used as input for the decision support modules after patient data has been transmitted from the DESIMS via FHIR.

\subsubsection{Production of recommendations}

The GL-DSS is launched from the DESIMS user interface. The user 


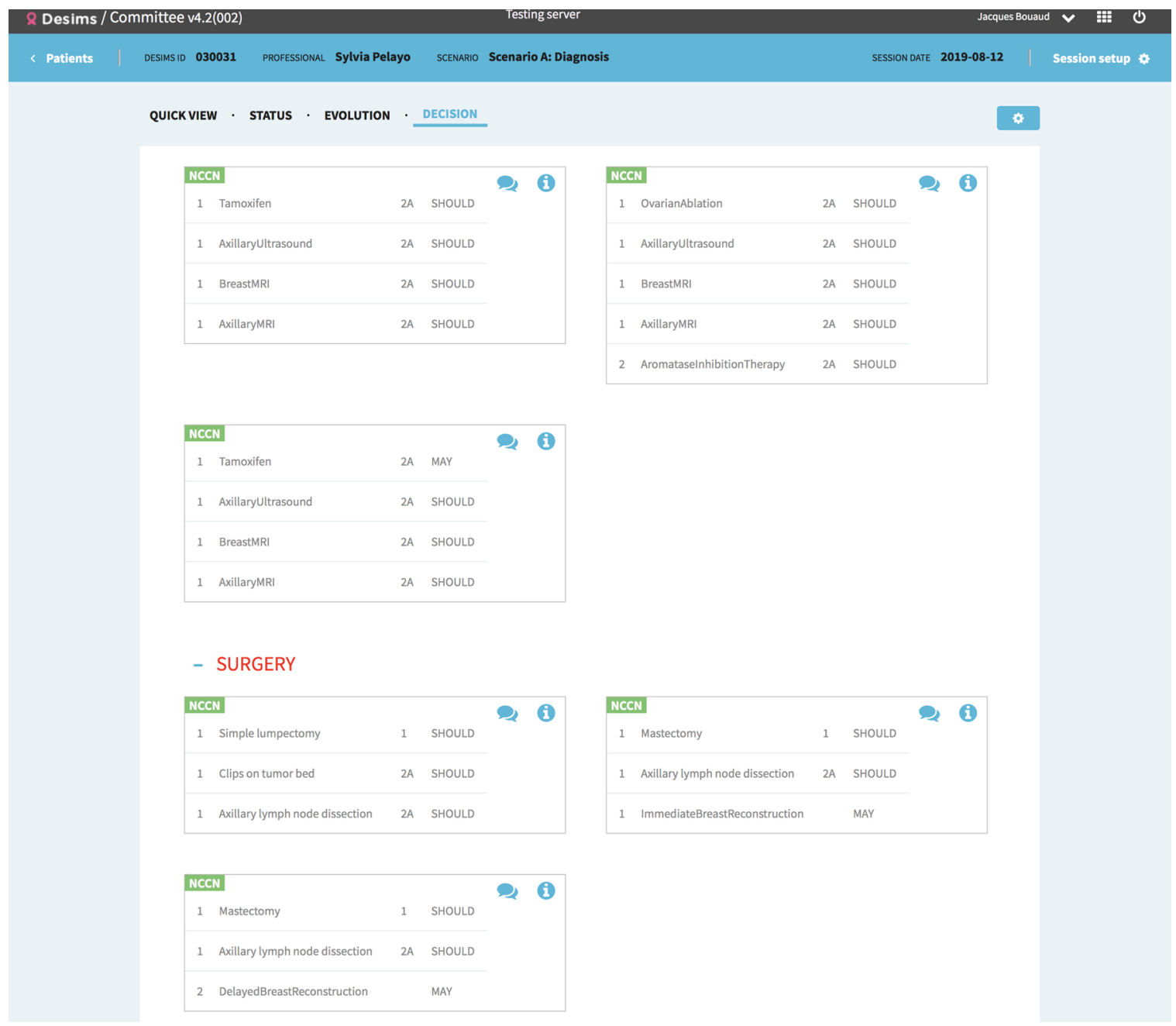

Fig. 15. Display of the recommended care plans in the DESIMS.

Table 1

Preliminary results of the clinical assessment of the GL-DSS by the three clinical pilot sites.

\begin{tabular}{llllll}
\hline & $\begin{array}{l}\text { \# TB } \\
\text { clinicians }\end{array}$ & $\begin{array}{l}\text { \# TB } \\
\text { sessions }\end{array}$ & \# Decisions & $\begin{array}{l}\text { Compliance } \\
\text { of } \mathrm{D}_{\text {without }}\end{array}$ & $\begin{array}{l}\text { Compliance } \\
\text { of } \mathrm{D}_{\text {with }}\end{array}$ \\
\hline ONK & 3 & 6 & 86 & $98.8 \%$ & $96.5 \%$ \\
ERE & 3 & 3 & 17 & $100.0 \%$ & $100.0 \%$ \\
AP-HP (GPEH) & 3 & $5(+3)$ & 35 & $100.0 \%$ & $97.1 \%$ \\
Total & 9 & $\begin{array}{l}14(+ \\
3)\end{array}$ & 138 & $99.3 \%$ & $97.1 \%$ \\
& & & & \\
\hline
\end{tabular}

may interactively choose the CPGs she wants the GL-DSS to consider for the decision support (NCCN, AP-HP, ONK). A number of recommendations are then returned and displayed to TB clinicians which may either decide to follow one of the suggestions or, if not, enter their final decision. For instance, when the GL-DSS is launched on the clinical case described above with NCCN CPGs, several different concurrent therapeutic options are provided, basically two surgeries and multiple options of systemic neo-adjuvant therapies. Fig. 14 provides an illustration of the XML recommendation nested structure returned by the GL-DSS and made of:
- At the first level, a recommended lumpectomy (with a conformance level "SHOULD") associated with a recommended axillary exploration, refined as an axillary lymph node dissection while the lumpectomy is complemented by the placement of clips on the tumor bed; - At the first level, a recommended mastectomy (SHOULD) associated with a recommended axillary exploration, refined as an axillary lymph node dissection, and complemented with an optional breast reconstruction (conformance level "MAY"), which can be refined into either an immediate breast reconstruction or a delayed breast reconstruction;

- The third option for this patient is a neo-adjuvant systemic therapy, which is refined into several recommended (SHOULD) or optional (MAY) chemotherapy protocols along with endocrine therapy, which can be refined as Tamoxifen therapy.

Finally, recommendations are displayed in the DESIMS user interface (see Fig. 15) at the most specific level by flattening the initial nested recommendation structure. From our example, the first surgery option is made of a block tagged with the guideline ID, i.e., "NCCN", where procedures (lumpectomy, clips on the tumor bed, and axillary lymph node dissection) are listed with their evidence level and conformance level. 


\subsection{Clinical evaluation}

A clinical evaluation of the DESIREE platform was performed in July 2019 by all the clinical partners of the project, Onkologikoa (ONK, a cancer center located in San Sebastian, Spain), ERESA (ERE, providing diagnostic imaging and radiotherapy, Valencia, Spain), and Assistance Publique - Hôpitaux de Paris (AP-HP, the first cancer care institution in Paris region and France, with the Georges Pompidou European Hospital, GPEH, as the pilot site). We report in this paper the preliminary results of the clinical evaluation of the sole GL-DSS prototype.

The GL-DSS was evaluated by three clinical pilot sites (ONK, ERE and GPEH). All sites selected a sample of past retrospective clinical cases previously discussed in real TBs without the GL-DSS and for which $\mathrm{D}_{\text {without }}$ decisions were recorded. The past clinical cases used were different from one clinical site to the other. For the evaluation, the past clinical cases were anonymized and re-discussed in close to real (simulated) TBs (meetings involved at least the three mandatory medical specialties for breast cancer decisions, a radiotherapist, a medical oncologist, and a surgeon) and $\mathrm{D}_{\text {with }}$ decisions were collectively made using the GL-DSS (each pilot site worked on its own past clinical cases). Prior to the beginning of the evaluation, a short user training video has been presented to TB clinicians to describe (i) the main functionalities of the whole DESIREE platform, with a focus on (ii) how to register a patient for a TB, (iii) how data describing clinical cases are organized in the DESIMS, and (iv) how the GL-DSS should be used.

Seventeen simulated TBs were organized (among which three encountered technical problems such as delays in response times for different reasons), each one included three clinicians, and a total of 138 $D_{\text {with }}$ decisions were recorded for 110 different patients. The compliance rate of $D_{\text {without }}$ decisions with GL-DSS recommendations was very high, i.e., $99.3 \%$ and the compliance rate of $\mathrm{D}_{\text {with }}$ decisions with GL-DSS recommendations remained at the same very high level (97.1 $\%)$. The few non-compliant decisions with the GL-DSS were mainly explained by some flaws in the interface (i.e., the DESIMS was actually displaying the recommendation finally decided by $\mathrm{TB}$ clinicians, but because there were too many recommendations, clinicians did not manage to retrieve them from the interface, or TB clinicians wanted to choose two recommendations but this was not allowed). The comparison of $D_{\text {without }}$ and $D_{\text {with }}$ decisions showed that clinicians modified their prescriptions 24 times out of 138 decisions when using the GL-DSS (i.e., in $17.4 \%$ of the cases). An external expert in the domain of breast cancer management assessed the quality of $D_{\text {without }}$ and $D_{\text {with }}$ decisions and established that $\mathrm{D}_{\text {with }}$ decisions were strictly better decisions than $\mathrm{D}_{\text {without }}$ decisions in 18 cases out of 24 (in $75 \%$ of the cases), e.g., "Endocrine therapy" without the GL-DSS and "Tamoxifen therapy" with the GL-DSS. A summary of the results is displayed in Table1.

At the end of the simulated TBs, we performed a qualitative analysis. TB clinicians were asked to complete the User Experience Questionnaire [49] and to answer some questions about the added value of the system, what they especially appreciated, what they especially disliked, what would be the barriers and the facilitators for using the system in the future during TBs. Qualitative data was also extracted from the recordings of TB sessions. Results showed a very positive evaluation of the GL-DSS from the clinicians regarding the attractiveness and ease of use of the system. For instance, Fig. 16 illustrates with a word cloud the clinicians' answers to the question "What did you especially appreciate in the GL-DSS and why?". Perceived as promising and easy to learn, the GL-DSS system caught the interest of clinicians who declared they were ready to use it in a daily practice if the system is extended to manage more "complex" patient cases. The current version was perceived as rather useful for non-expert centers.

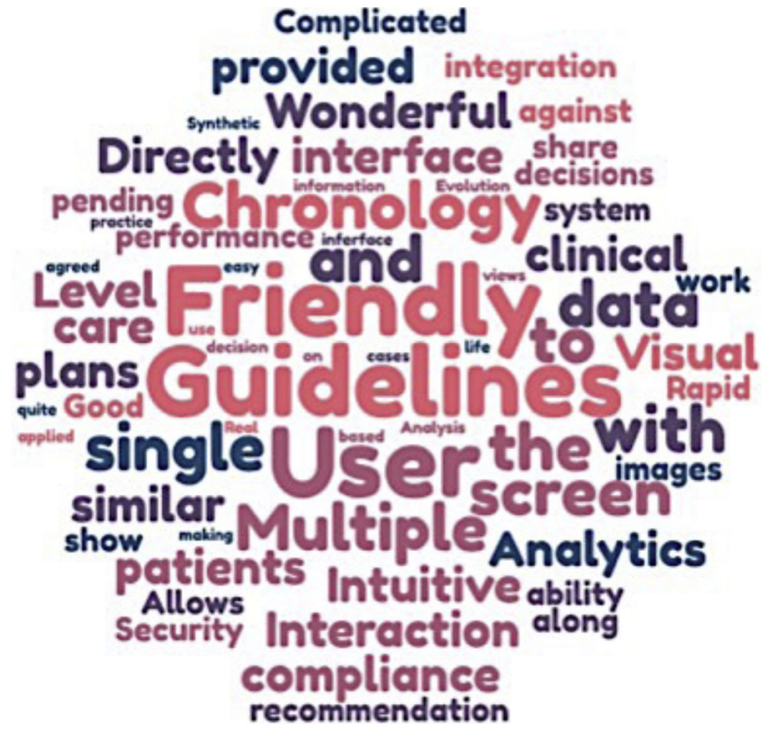

Fig. 16. Word cloud synthesizing answers to the question "What did you especially appreciate in the GL-DSS and why?".

\section{Discussion and conclusion}

The GL-DSS of the DESIREE project is a guideline-based CDSS applied to the management of breast cancer patients. Supporting the implementation of CPGs by CDSSs has a long history. The first CDSSs applied to the management of breast cancer patients date back to 1986 [50]. These systems were expert systems with knowledge bases formalizing the expertise of clinicians engaged in decision-making tasks. Since then, various CDSS prototypes have been developed for the management of breast cancer. Most of them are guideline-based systems to support the decisions of TB clinicians, e.g., MATE [24], OncoCure [51], and OncoDoc [52,25]. More recently, systems such as IBM's Watson for Oncology [53] or the Oncology Expert Advisor [54] seek to build oncology decision support tools using artificial intelligence components trained on data extracted from scientific literature (querying bibliographic databases such as PubMed) and retrospective TB decisions. However, results are controversial and IBM's Watson for Oncology has come under fire for not delivering on expectations to provide state-of-the-art personalized treatment for cancer patients and for producing advice that is "unsafe and incorrect" [55,56]. To the authors' knowledge, if breast cancer CDSSs have been assessed on retrospective real patient data with high rates of agreement between TB decisions and the CDSS's recommendations (93.2 \%, 85.2 \%, $93.4 \%$, and $93 \%$ for Mate [24], Oncocure [51], OncoDoc [25], and Watson for Oncology [53], resp.), a few oncology CDSSs have been actually routinely used during TB meetings except OncoDoc2 [23], and only a small number demonstrated that they did improve the compliance of TB decisions with CPGs (e.g., Oncodoc2 showed to have a compliance rate of $91.7 \%$ [25]). A recent systematic review [57] showed that few studies have assessed the outcomes of CDSSs for oncology practice, and has concluded on the critical need for CDSSs development and evaluation. Aiming at developing a breast cancer CDSS improving existing systems, the GL-DSS of the DESIREE project is in line with these two objectives.

Different formalisms have been used to represent CPG contents in breast cancer guideline-based CDSSs. For instance, Mate [24] uses the PROforma language based on the CREDO software platform [58]. In Oncocure [51], CPGs are encoded using Asbru that represents clinical guidelines and protocols as time-oriented skeletal plans [59]. OncoDoc [25] proposes a documentary approach to breast cancer decision 
support where the user may navigate through a knowledge base structured as a decision tree to get the best patient-specific recommendations. Using semantic web technologies, Abidi et al. [60] developed a web-based execution engine to combine the ontology representing CPGs and associated domain knowledge, and the patient ontology describing the patient's state. More generally, numerous dedicated formalisms have been proposed to translate free-text CPGs into computer-interpretable guidelines [61,62]. In the GL-DSS of the DESIREE project, we have used the Natural Rule Language [41] as the formalism to specify rules in order to represent guideline knowledge. Inspired by OCL (Object Constraint Language), and originally designed as a language for testing the validity of data models [63], NRL provides a syntax for writing logical expressions consistent with the data model components, independently of the specification of an execution engine. NRL rules are then transformed in N3 notation, the target computerinterpretable guideline formalism to be used by the semantic inference engine, EYE, combining ontological reasoning and rules. Using tools from the semantic web domain as a base could have led to some kind of solution based on SWRL rules associated to a classical OWL reasoning engine to produce inferences. However, because of the lack of expressiveness of the latter, the impossibility to deal with non-monotonicity and negation within the open world assumption and the degraded performances obtained on real-life applications with these techniques, we chose to adopt an alternative solution with Euler/EYE which does not have these limitations. Besides, this approach allows a reasoning process able to produce therapeutic treatment recommendations with varying levels of abstraction for the patients whose clinical profiles can also be described at varying levels of abstraction. The design of the BCKM was done in the context of the development of a research prototype with the strong constraint of representing real data from the DESIMS (that relies on a relational model). We chose to use the EAV data model, which is simple but generic and flexible enough to model biomedical data [36-38]. In addition, we chose to explain each element of the EAV model as classes and not exploit the OWL possibilities where attributes could have been represented by DataProperties for primitive types and ObjectProperties for hierarchical types. This class representation allows to import concepts / classes from other ontologies and to give them any role in the BCKM: entity, attribute, or value. Thus, we have imported many concepts and hierarchies from the NCI thesaurus to the BCKM using the Flat OWL Editor tool [39]. It should be noted that in this case, the link between the NCI thesaurus and its cloned classes is not dynamically conserved as it can be the case with a tool such as OntoFox [64] but it is static with the NCI_CODE kept as annotation. However, we did not look for the dynamic preservation of the import link, first because we did not consider that the structuring of the NCI thesaurus was satisfactory for our application (the organization of some hierarchies had to be reviewed manually), and second, the coding of existing data for certain versions of the BCKM and its concepts might not be able to withstand the evolutions of the BCKM, which should be controlled. In the current state, the quality of the BCKM is quite satisfactory since it allows to represent information, both DESIMSprovided patient data and guideline knowledge, in a way that enables decision-making and automatic production of recommendations with a good level of performance, as established by the technical and clinical validation steps of the GL-DSS.

Interoperability of medical data is essential to improve care quality and efficiency, and there are multiple standards available for clinical data exchange, e.g., the Consolidated Clinical Document Architecture (C-CDA) and the Fast Health Interoperability Resources (FHIR) maintained by Health Level 7 (HL7). Unfortunately, the data collected in health information tools is often in a non-standard, non-structured, or even non-coded (text) form, resulting in a lack of interoperability. The main issue for CDSSs is to get patient data originally input into electronic health records (EHRs). For instance, MATE [24] contains its own data entry facilities for its stand-alone EHR. Concerning OncoDoc [25] and Abidi's work [59], all patient data are entered as needed in the decision process and there is no linking with an EHR, while OncoCure [51] is integrated into an existing EHR used at the point of care, relieving the user from data entry. In the DESIREE project, the DESIREE Information Management System (DESIMS) acts as an EHR where patient data is stored in a dedicated database. However, the DESIMS was designed prior to the DESIREE project, with an information model different from the one adopted in the BCKM and an equally different terminology repository. While the use of FHIR has made it possible to solve the syntactical dimension of interoperability, semantic interoperability required manual alignment between the terms used in the DESIMS repository and the BCKM concepts. This finding calls for the sharing of termino-ontological references, but also models of clinical information [65], possibly dedicated to a particular area such as cancer [66]. In addition, beyond classic semantic interoperability issues, TB decisions are the result of collective discussions and, although based on CPGs, they do take into account a variety of "holistic parameters" [51] that may not be directly represented in EHRs, such as complex, implicit, hard to codify knowledge, or individual patient preferences. No holistic parameters were included in the DESIMS data model whereas some of them were part of the BCKM. As a consequence, the reasoning process performed by the GL-DSS was reduced because some holistic patient data were missing due to the DESIMS filter. Since we chose to explain each element of the EAV model as classes, we have started to experiment another solution to reinforce interoperability between EHR-like components used to collect patient data and the GL-DSS. Indeed, having classes to represent each element of the EAV model makes the hasRange relationship between a ModelAttribute class and a ModelValue class generic and allows to process it uniformly regardless of the value type. This feature has been exploited for the automatic construction of input forms from the ontological BCKM, to be used as integrated EHR-like interfaces [67]. Beyond re-enforcing interoperability, this has also been used to explore the whole potential of the GL-DSS reasoning process on non-simple clinical cases including holistic parameters not considered by the DESIMS.

All published guideline-based breast cancer CDSSs rely on the modeling and implementation of a single CPG applied to a unique pathology, the management of breast cancer. Since computer-interpretable CPGs are built from the translation of free-text guidelines, they import some of the natural language weaknesses, and even with only one disease, guideline-based decision support systems have to deal with intra-CPGs inconsistencies. This difficulty is even more important when handling the concurrent application of CPGs for different diseases in order to manage patients with comorbidities. Galopin et al., [68] have implemented an ontological reasoning process to allow for the flexibility necessary to deal with patients suffering from both hypertension and type 2 diabetes. Wilk et al. [69], have proposed a framework based on first order logic to represent CPGs and to mitigate possible adverse interactions (drug-drug or drug-disease) between the recommendations provided by the different CPGs. Abidi et al., [70] proposed the CPG integration framework COMET to manage multiple CPGs using comorbidity management procedures based on the input of domain experts. In these cases, CPGs are reconciled on the basis of competition and the goal is to select the recommendations that are "best suited" to the patient complexity. In the DESIREE project, we chose to use three CPGs (NCCN, AP-HP, and ONK) to support the decision of the clinical partners of the project with the provision of their local guidelines, but also to offer to all of them the possibility to get recommendations from international CPGs and to extend the clinical coverage of the GL-DSS [71]. Indeed, we know that there are some knowledge gaps in CPG contents that lead to the silence of the CDSS for the specific profiles not covered by CPGS. Thus, the bet was that even if we used contemporary CPGs versions (all CPGs embedded within the GL-DSS were published in 2017), knowledge gaps of some CPGs could be filled in by the others. This has been confirmed in the special case of NCCN and AP-HP CPGs where the silence of one CPG was resolved by the other CPG to allow the GL-DSS to provide recommendations in $21 \%$ of the cases [72]. As 
opposed to the management of multiple CPGs in case of polymorbid patients, the aim was to reconcile breast cancer CPGs on the basis of complementarity. In addition, reconciliation solutions have been developed within the decision rules (complement and refine). But we have to develop a post treatment of the recommendations provided to solve the conflicts and build care plans from atomic recommendations while benefiting from the complementarity of CPGS.

The GL-DSS module of the DESIREE project aims at providing decision support at different levels of abstraction, allowing for flexibility in the reasoning process. We presented the developments we made and justified our choice to use semantic web tools. We constructed a single domain ontology used as a conceptual and terminological structure to provide a data model and a knowledge model, used for reasoning and decision support. The resulting BCKM plays a pivotal role in data and knowledge management. Other studies aim at combining information models and ontologies [73,74]. The GL-DSS of the DESIREE project has been implemented in the basis of three CPGs (NCCN, AP-HP, and ONK). It has been assessed during simulated TBs in three pilot sites on 138 decisions. It appeared that the clinical cases used for the clinical validation were simple cases with a high rate of TB decisions compliance with CPGs. This high rate was conserved when using the system to solve the same clinical cases. However, the quality of TB decisions was improved when using the system for 18 clinical cases out of the 24 where TB clinicians changed their decision. This result has to be confirmed with a larger study involving complex clinical cases in AP-HP real-life TBs. Prior to this evaluation, our future works would be to develop the prerequisites to achieve a true interoperability between the AP-HP EHR system (Orbis from $\mathrm{Agfa}^{3}$ ), to implement the reconciliation of CPGs to solve decisional conflicts, and to build care plans from atomic recommendations. We especially would have to develop an algebra of atomic recommendations conformance levels to compute the conformance level of the inferred care plans.

\section{Declaration of Competing Interest}

The authors declare that there are no conflicts of interest.

\section{Acknowledgments}

The DESIREE project was funded by the European Union's Horizon 2020 research and innovation programme under grant agreement no. 690238 (H2020 PHC-30-2015). The authors would like to thank all the partners of the DESIREE project, especially the clinicians from Onkologikoa, Ander Urruticoechea Ribate, Jon Belloso Linacisoro, and Gerardo Cajaraville Ordonana, as well as the participating members of Vicomtech, among which Nekane Larburu, Naiara Muro, and Ivan Macia, and the participating members of Bilbomatica, among which Amaia Ugarriza Pinedo, Ander Elzo Zarraga, and Roberto Alvarez Sanchez.

\section{References}

[1] Elsamany S, Abdullah S. Triple-negative breast cancer: future prospects in diagnosis and management. Med Oncol 2014.31(2):834.

[2] Sackett DL, Rosenberg WM, Gray JA, Haynes RB, Richardson WS. Evidence based medicine: what it is and what it isn't. BMJ 1996;312(7023):71-2.

[3] Haynes RB, Devereaux PJ, Guyatt GH. Physicians' and patients' choices in evidence based practice. BMJ 2002;324(June (7350)):1350.

[4] Hébert-Croteau N, Brisson J, Latreille J, Rivard M, Abdelaziz N, Martin G. Compliance with consensus recommendations for systemic therapy is associated with improved survival of women with node-negative breast cancer. J Clin Oncol 2004;22(18):3685-93.

[5] Varga D, Wischnewsky M, Atassi Z, Wolters R, Geyer V, Strunz K, et al. Does guideline-adherent therapy improve the outcome for early-onset breast cancer patients? Oncology 2010;78(3-4):189-95.

[6] Wöckel A, Kurzeder C, Geyer V, Novasphenny I, Wolters R, Wischnewsky M, et al.

\footnotetext{
${ }^{3}$ https://global.agfahealthcare.com/main/hospital-it/orbis/
}

Effects of guideline adherence in primary breast cancer-a 5-year multi-center cohort study of 3976 patients. Breast 2010;19(2):120-7.

[7] Schwentner L, Wolters R, Koretz K, Wischnewsky MB, Kreienberg R, Rottscholl R, et al. Triple-negative breast cancer: the impact of guideline-adherent adjuvant treatment on survival-a retrospective multi-centre cohort study. Breast Cancer Res Treat 2012;132(3):1073-80.

[8] Wolters R, Wischhusen J, Stüber T, Weiss CR, Krockberger M, Bartmann C, et al Guidelines are advantageous, though not essential for improved survival among breast cancer patients. Breast Cancer Res Treat 2015;152(2):357-66.

[9] Andreano A, Rebora P, Valsecchi MG, Russo AG. Adherence to guidelines and breast cancer patients survival: a population-based cohort study analyzed with a causal inference approach. Breast Cancer Res Treat 2017;164(1):119-31.

[10] Hsieh MC, Zhang L, Wu XC, Davidson MB, Loch M, Chen VW. Population-based study on cancer subtypes, guideline-concordant adjuvant therapy, and survival among women with stage I-III breast cancer. J Natl Compr Canc Netw 2019;17(6):676-86.

[11] Kreienberg R, Wöckel A, Wischnewsky M. Highly significant improvement in guideline adherence, relapse-free and overall survival in breast cancer patients when treated at certified breast cancer centres: an evaluation of 8323 patients. Breast 2018;40:54-9.

[12] Lebeau M, Mathoulin-Pélissier S, Bellera C, Tunon-de Lara C, Daban A, Lipinski F, et al. Breast cancer care compared with clinical guidelines: an observational study in France. BMC Public Health 2011:11:45.

[13] Patkar V, Acosta D, Davidson T, Jones A, Fox J, Keshtgar M. Cancer multidisciplinary team meetings: evidence, challenges, and the role of clinical decision support technology. Int J Breast Cancer 2011;2011:831605.

[14] Kesson EM, Allardice GM, George WD, Burns HJ, Morrison DS. Effects of multidisciplinary team working on breast cancer survival: retrospective, comparative, interventional cohort study of 13722 women. BMJ 2012;344. e2718.

[15] van Hoeve J, de Munck L, Otter R, de Vries J, Siesling S. Quality improvement by implementing an integrated oncological care pathway for breast cancer patients. Breast 2014;23(4):364-70.

[16] Brar SS, Hong NL, Wright FC. Multidisciplinary cancer care: Does it improve outcomes? J Surg Oncol 2014;110(5):494-9.

[17] Keating NL, Landrum MB, Lamont EB, Bozeman SR, Shulman LN, McNeil BJ. Tumor boards and the quality of cancer care. J Natl Cancer Inst 2013;105(2):113-21.

[18] Johnson CE, Slavova-Azmanova N, Saunders C. Development of a peer review framework for cancer multidisciplinary meetings. Intern Med J 2017;47(5):529-35.

[19] Rosell L, Alexandersson N, Hagberg O, Nilbert M. Benefits, barriers and opinions on multidisciplinary team meetings: a survey in Swedish cancer care. BMC Health Serv Res 2018;18(1):249.

[20] Jaspers MW, Smeulers M, Vermeulen H, Peute LW. Effects of clinical decision support systems on practitioner performance and patient outcomes: a synthesis of high-quality systematic review findings. J Am Med Inform Assoc 2011:18(3):327-34

[21] Roshanov PS, Misra S, Gerstein HC, Garg AX, Sebaldt RJ, Mackay JA, et al. Computerized clinical decision support systems for chronic disease management: a decision-maker-researcher partnership systematic review. Implement Sci $2011 ; 6: 92$.

[22] Van de Velde S, Heselmans A, Delvaux N, Brandt L, Marco-Ruiz L, Spitaels D, et al. A systematic review of trials evaluating success factors of interventions with computerised clinical decision support. Implement Sci 2018;13(August (1)):114.

[23] Séroussi B, Bouaud J, Gligorov J, Uzan S. Supporting multidisciplinary staff meet ings for guideline-based breast cancer management: a study with OncoDoc2. AMIA Annu Symp Proc 2007(October):656-60.

[24] Patkar V, Acosta D, Davidson T, Jones A, Fox J, Keshtgar M. Using computerised decision support to improve compliance of cancer multidisciplinary meetings with evidence-based guidance. BMJ Open 2012;2(3). e000439.

[25] Séroussi B, Laouénan C, Gligorov J, Uzan S, Mentré F, Bouaud J. Which breast cancer decisions remain non-compliant with guidelines despite the use of computerized decision support? Br J Cancer 2013;109(5):1147-56.

[26] Rampun A, Morrow PJ, Scotney BW, Winder J. Breast density classification using multiresolution local quinary patterns in mammograms. Med Image Understanding Anal Proc 2017:365-76.

[27] Rampun A, Scotney BW, Morrow PJ, Wang H, Winder J. Segmentation of breast MR images using a generalised 2D mathematical model with inflation and deflation forces of active contours. Artif Intell Med 2019;97(June):44-60.

[28] Rampun A, Scotney BW, Morrow PJ, Wang H. Breast mass classification in mammograms using ensemble convolutional neural networks. 2018 IEEE 20th International Conference on E-Health Networking, Applications and Services (Healthcom) 2020.

[29] Salmon R, Nguyen T, Lesage AC, Bass B, Garbey M. Clinical study and multiscale modeling to predict the esthetic outcome of Breast Conservative Therapy. 2017. https://doi.org/10.13140/RG.2.2.35635.94245.

[30] Séroussi B, Lamy JB, Muro N, Larburu N, Sekar BD, Guézennec G, et al. Experiencebased, and case-based approaches to enrich decision support for the management of breast cancer patients in the DESIREE project. Stud Health Technol Inform 2018;255:190-4.

[31] Shiffman RN, Michel G, Essaihi A, Thornquist E. Bridging the guideline implementation gap: a systematic, document-centered approach to guideline implementation. J Am Med Inform Assoc 2004;11(5):418-26.

[32] Muro N, Larburu N, Bouaud J, Belloso J, Cajaraville G, Urruticoechea A, et al Augmenting guideline knowledge with non-compliant clinical decisions: experience-based decision support. KES-InMed 2018 2017: innovation in medicine and healthcare. 2017. p. 217-26.

[33] Sekar BD, Lamy JB, Larburu N, Séroussi B, Guézennec G, Bouaud J, et al. Case-based 
decision support system for breast cancer management. Int J Comput Int 2018;12:28-38.

[34] Bouaud J, Guézennec G, Séroussi B. Combining the generic entity-attribute-value model and terminological models into a common ontology to enable data integration and decision support. Stud Health Technol Inform 2018;247:541-5.

[35] HL7.org. FHIR Release 3 (STU; v3.0.2), http://hl7.org/fhir/STU3/. [last accessed Jul 10, 2020].

[36] Nadkarni PM, Marenco L, Chen R, Skoufos E, Shepherd G, Miller P. Organization of heterogeneous scientific data using the EAV/CR representation. J Am Med Inform Assoc 1999;6(6):478-93.

[37] Löper D, Klettke M, Bruder I, Heuer A. Enabling flexible integration of healthcare information using the entity-attribute-value storage model. Health Inf Sci Syst 2013;1:9.

[38] Khan O, Lim Choi Keung SN, Zhao L, Arvanitis TN. A hybrid EAV-Relational model for consistent and scalable capture of clinical research data. Stud Health Technol Inform 2014;202:32-5.

[39] Despres S, G. Guézennec. Flat OWL Editor : un outil utilisant des feuilles de calcul pour découpler les tâches des acteurs impliqués dans la gestion d'une ontologie. Terminologie \& Ontologie: Théories et Applications, Actes de la conference TOTh 2017. Chambéry, France: Université Savoie Mont Blanc; 2017. p. 181-94.

[40] Gennari J, Musen M, Fergerson R, Grosso W, Crubézy M, Eriksson H, et al. The evolution of Protégé: an environment for knowledge-based systems development. Int J Hum Comput Stud 2003;58:89-123.

[41] Nentwich C, James R. Natural rule language (NRL) specification 1.4.0. 2010 [last accessed Jul 10, 2020]. http://nrl.sourceforge.net/spec/.

[42] Breast cancer. Version 3.2017. NCCN clinical practice guidelines in oncology (NCCN guidelines ${ }^{\circledR}$ ). 2017.

[43] AP-HP. Référentiel cancer du sein, mars 2016. Référentiels de l' AP-HP [last accessed Jul 10, 2020]. https://www.aphp.fr/sites/default/files/referentiel_cancers du_sein - juin_2016_1.pdf.

[44] Shahar Y, Young O, Shalom E, Mayaffit A, Moskovitch R, Hessing A, et al. The Digital electronic Guideline Library (DeGeL): a hybrid framework for representation and use of clinical guidelines. Stud Health Technol Inform 2004;101:147-51.

[45] Meditskos G, Bassiliades N. Rule-based OWL ontology reasoning systems: implementations, strengths and weaknesses. 2009. https://doi.org/10.4018/978-160566-402-6.ch006.

[46] Verborgh R, De Roo J. Drawing conclusions from linked data on the web: the EYE reasoner. IEEE Softw 2015;32(3):23-7.

[47] Berners-Lee T, Connolly D, Kagal L, Scharf Y, Hendler J. N3Logic: a logical framework for the World Wide Web. Theory Pract Log Program 2008;8(3):249-69.

[48] HL7.org. Hl7 standard: Clinical decision support knowledge artifact specification, release 1.3. 2015 [last accessed Jul 10, 2020]. http://www.hl7.org/implement/ standards/product_brief.cfm?product_id $=337$.

[49] Laugwitz B, Schrepp M, Held T. Holzinger A, editor. Construction and evaluation of a user experience questionnaire. 2008. p. 63-76. USAB 2008, LNCS 5298.

[50] Gaglio S, Ruggiero C, Spinelli G, Bonadonna G, Valagussa P, BREASTCAN C Nicolini,. An expert system for postoperative breast cancer therapy. Comput Biomed Res 1986;19:445-6.

[51] Eccher C, Seyfang A, Ferro A. Implementation and evaluation of an Asbru-based decision support system for adjuvant treatment in breast cancer. Comput Methods Programs Biomed 2014;117(2):308-21.

[52] Séroussi B, Bouaud J, Antoine EC. OncoDoc: a successful experiment of computersupported guideline development and implementation in the treatment of breast cancer. Artif Intell Med 2001;22(1):43-64.

[53] Lee WS, Ahn SM, Chung JW, Kim KO, Kwon KA, Kim Y, et al. Assessing concordance with Watson for Oncology, a cognitive computing decision support system for colon cancer treatment in Korea. JCO Clin Cancer Inform 2018;2:1-8.

[54] Simon G, DiNardo CD, Takahashi K, Cascone T, Powers C, Stevens R, et al. Applying artificial intelligence to address the knowledge gaps in cancer care. Oncologist 2018;24(6):772-82.

[55] Ross C, Swetlitz I. IBM's Watson supercomputer recommended "unsafe and incorrect" cancer treatments, internal documents show. STAT. 2018 July 25 Available at www.statnews.com/wp-content/uploads/2018/09/IBMs-Watsonrecommended-unsafe-and-incorrect-cancer-treatments-STAT.pdf. [last accessed Jul $10,2020]$

[56] Hernandez D, Greenwald T. IBM has a Watson dilemma. The Wall Street Journal; 2018 August 11, Available at www.wsj.com/articles/ibm-bet-billions-that-watsoncould-improve-cancer-treatment-it-hasnt-worked-1533961147. [last accessed Jul $10,2020]$.

[57] Pawloski PA, Brooks GA, Nielsen ME, Olson-Bullis BA. A systematic review of clinical decision support systems for clinical oncology practice. J Natl Compr Canc Netw 2019;17(April (4)):331-8.

[58] Fox J, Chronakis I, Glasspool D, Patkar V, Peleg M, South M, et al. Integrating clinical decision support and smart guidelines: a new approach to evidence-based medicine. Junior Doctor J. 2013;3http://is.haifa.ac.il/ morpeleg/pubs/D38.pdf.

[59] Shahar Y, Miksch S, Johnson P. The Asgaard project: a task-specific framework for the application and critiquing of time-oriented clinical guidelines. Artif Intell Med 1998;14(1-2):29-51.

[60] Abidi SR, Abidi SR, Hussain S, Shepherd M. Ontology-based modeling of clinical practice guidelines: a clinical decision support system for breast cancer follow-up interventions at primary care settings. Stud Health Technol Inform 2007;129(2):845-9.

[61] Peleg M. Computer-interpretable clinical guidelines: a methodological review. J Biomed Inform 2013;46(4):744-63.

[62] de Clercq P, Kaiser K, Hasman A. Computer-interpretable guideline formalisms. In ten Teije A, Miksch S, Lucas P, editors. Computer-based medical guidelines and protocols: a primer and current trends, vol. 139 of studies in health technology and Informatics. IOS Press; 2008. p. 22-43.

[63] Boscá D, Maldonado JA, Moner D, Robles M. Automatic generation of computable implementation guides from clinical information models. J Biomed Inform 2015;55:143-52.

[64] Xiang Z, Courtot M, Brinkman RR, Ruttenberg A, OntoFox: Y He,. Web-based support for ontology reuse. BMC Res Notes 2010;3(175).

[65] Becnel LB, Hastak S, Ver Hoef W, Milius RP, Slack M, Wold D, et al. BRIDG: a domain information model for translational and clinical protocol-driven research. $J$ Am Med Inform Assoc 2017;24(5):882-90.

[66] Hochheiser H, Castine M, Harris D, Savova G, Jacobson RS. An information mode for computable cancer phenotypes. BMC Med Inform Decis Mak 2016;16(1):121.

[67] Sadki F, Bouaud J, Guézennec G, Séroussi B. Semantically structured web form and data storage: a generic ontology-driven approach applied to breast cancer. Stud Health Technol Inform 2018;255:205-9.

[68] Galopin A, Bouaud J, Pereira S, Séroussi B. An ontology-based clinical decision support system for the management of patients with multiple chronic disorders. Stud Health Technol Inform 2015;216:275-9.

[69] Wilk S, Michalowski W, Michalowski M, Farion K, Hing MM, Mohapatra S. Mitigation of adverse interactions in pairs of clinical practice guidelines using constraint logic programming. J Biomed Inform 2013;46(2):341-53.

[70] Abidi S. A, Knowledge-modeling approach to integrate multiple clinical practice guidelines to provide evidence-based clinical decision support for managing comorbid conditions. J Med Syst 2017;41:193.

[71] Séroussi B, Guézennec G, Lamy JB, Muro N, Larburu N, Sekar B, et al. Reconciliation of multiple guidelines for decision support: a case study on the multidisciplinary management of breast cancer within the DESIREE project. AMIA Annu Symp Proc 2017:1527-36.

[72] Séroussi B, Prébet C, Ngo C, Teixeira L, Guézennec G, Bouaud J. Using the recommendations inferred by a decision support system to compare breast cancer clinical practice guidelines. Stud Health Technol Inform 2019;258:60-4.

[73] Ethier JF, Curcin V, Barton A, McGilchrist MM, Bastiaens H, Andreasson A, et al. Clinical data integration model. Core interoperability ontology for research using primary care data. Methods Inf Med 2015;54(1):16-23.

[74] Martínez-Costa C, Schulz S. Validating EHR clinical models using ontology patterns. J Biomed Inform 2017;76:124-37. 